\title{
A THREE-FIELD AUGMENTED LAGRANGIAN FORMULATION OF UNILATERAL CONTACT PROBLEMS WITH COHESIVE FORCES*
}

\author{
David Doyen ${ }^{1},{\text { Alexandre } \text { Ern }^{2} \text { And Serge Piperno }}^{2}$
}

\begin{abstract}
We investigate unilateral contact problems with cohesive forces, leading to the constrained minimization of a possibly nonconvex functional. We analyze the mathematical structure of the minimization problem. The problem is reformulated in terms of a three-field augmented Lagrangian, and sufficient conditions for the existence of a local saddle-point are derived. Then, we derive and analyze mixed finite element approximations to the stationarity conditions of the three-field augmented Lagrangian. The finite element spaces for the bulk displacement and the Lagrange multiplier must satisfy a discrete inf-sup condition, while discontinuous finite element spaces spanned by nodal basis functions are considered for the unilateral contact variable so as to use collocation methods. Two iterative algorithms are presented and analyzed, namely an Uzawa-type method within a decompositioncoordination approach and a nonsmooth Newton's method. Finally, numerical results illustrating the theoretical analysis are presented.
\end{abstract}

Mathematics Subject Classification. 65N30, 65K10, 74S05, 74M15, 74R99.

Received December 23, 2008. Revised July 27, 2009.

Published online January 27, 2010.

\section{INTRODUCTION}

The purpose of this work is to analyze augmented Lagrangian methods for solving static unilateral contact problems with cohesive forces. Problems of this kind arise in fracture mechanics, such as crack initiation and growth in brittle and ductile materials as well as delamination of composite materials $[5,14]$. Unilateral contact problems without cohesive forces have been widely studied from both theoretical and numerical standpoints; see, for instance, $[16,20]$. They can be formulated as the minimization of a convex functional or, equivalently, as a monotone variational inequality. The presence of cohesive forces in addition to the unilateral contact makes the functional to be minimized possibly nonconvex or, equivalently, the operator in the variational inequality possibly non-monotone. This complicates substantially the problem.

Consider a prototypical unilateral contact problem with cohesive forces, as illustrated in Figure 1. The domain $\Omega \subset \mathbb{R}^{d}(d=2$ or $d=3$ ) represents a deformable body. The material is assumed to be linear isotropic

\footnotetext{
Keywords and phrases. Unilateral contact, cohesive forces, augmented Lagrangian, mixed finite elements, decompositioncoordination method, Newton's method.

* This work was partially supported by EDF RED. The first author was supported by a CIFRE Ph.D. fellowship.

1 EDF R\&D, 1 avenue du Général de Gaulle, 92141 Clamart Cedex, France. david.doyen@edf.fr

2 Université Paris-Est, CERMICS, École des Ponts, 77455 Marne-la-Vallée Cedex 2, France. ern@cermics.enpc.fr; piperno@cermics.enpc.fr
} 


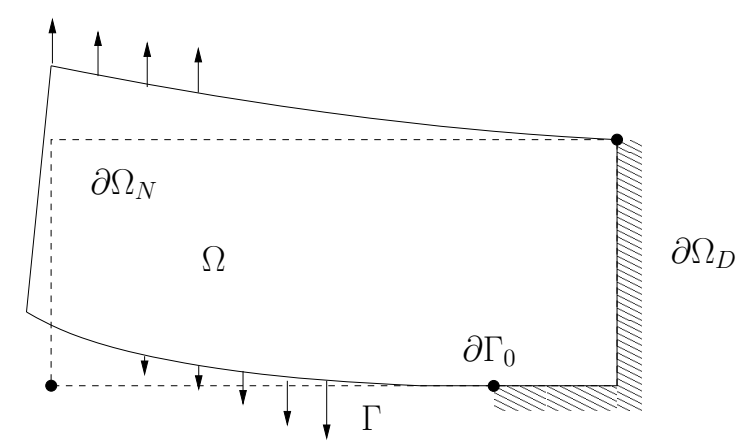

FiguRE 1. Example of unilateral contact problem with cohesive forces.
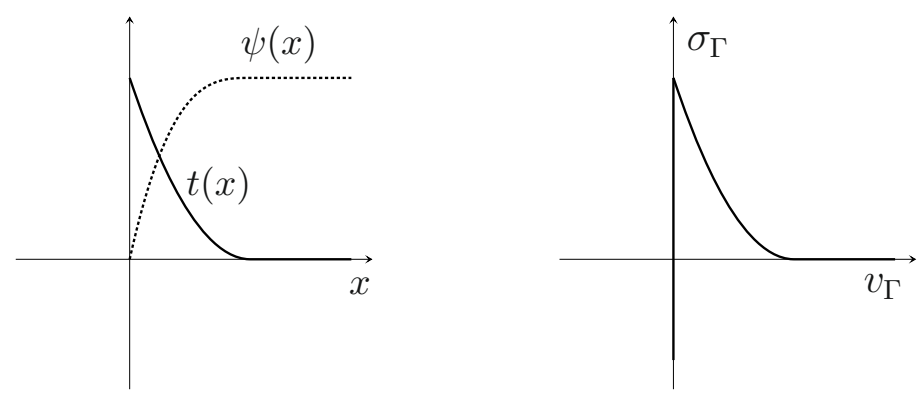

Figure 2. Example of cohesive law.

elastic, with Lamé coefficients $\lambda$ and $\mu$. Let $u: \Omega \rightarrow \mathbb{R}^{d}$ be the displacement field. The linearized strain tensor and stress tensor, $\epsilon(u): \Omega \rightarrow \mathbb{R}^{d, d}$ and $\sigma(u): \Omega \rightarrow \mathbb{R}^{d, d}$, are respectively defined as

$$
\epsilon(u)=\frac{1}{2}\left(\nabla u+\nabla u^{T}\right) \quad \text { and } \quad \sigma(u)=\lambda \operatorname{tr} \epsilon(u) I+2 \mu \epsilon(u) .
$$

An external load $f$ is applied to the body. The boundary $\partial \Omega$ is partitioned into three disjoint open subsets $\partial \Omega_{D}, \partial \Omega_{N}$, and $\Gamma$ (the measure of $\partial \Omega_{D}$ is supposed to be positive). An homogeneous Dirichlet condition and a Neumann condition are prescribed on $\partial \Omega_{D}$ and $\partial \Omega_{N}$, respectively. The normal load on $\partial \Omega_{N}$ is denoted by $g$.

On $\Gamma$, we impose a unilateral contact condition with cohesive forces. The cohesive forces depend on the displacement on $\Gamma$. The present model belongs to the class of so-called cohesive zone models; see, e.g., [5,14]. For the sake of simplicity, we restrict ourselves to the case where the cohesive forces are normal and depend only on the normal displacement. Let $n$ be the outward normal to $\Omega$ and let $v_{\Gamma}:=-\left.v\right|_{\Gamma} \cdot n$ and $\sigma_{\Gamma}:=\left.n \cdot \sigma\right|_{\Gamma} \cdot n$ respectively denote the normal displacement and the normal stress on $\Gamma$. Then, (i) $v_{\Gamma}$ cannot be negative; (ii) if $v_{\Gamma}$ is zero, $\sigma_{\Gamma}$ must be lower than a yield $\sigma_{c}$; and (iii) if $v_{\Gamma}$ is positive, $\sigma_{\Gamma}$ obeys the cohesive law $\sigma_{\Gamma}=t\left(v_{\Gamma}\right)$. Hence, the cohesive law is a function $t: \mathbb{R}^{+} \rightarrow \mathbb{R}$, and we define a cohesive energy $\psi: \mathbb{R}^{+} \rightarrow \mathbb{R}$ such that $\psi^{\prime}=t$ and, say, $\psi(0)=0$. For later convenience, we extend the domain of $\psi$ to $\mathbb{R}$ by setting for $s \geq 0, \psi(-s)=-\psi(s)$. There is a large variety of cohesive laws. Their common feature is a softening behavior: when the displacement increases, the cohesive force decreases. Consequently, the boundary condition is non-monotone and the cohesive energy is nonconvex. Typical functions $t$ and $\psi$ are represented on the left part of Figure 2. The boundary condition is represented on the right part of Figure 2. 
Let $V$ and $H$ be function spaces on $\Omega$ and $\Gamma$, respectively, defined in Section 2 below. Consider the functionals

$$
\begin{aligned}
& W: V \ni v \longmapsto W(v):=\frac{1}{2} \int_{\Omega} \sigma(v): \epsilon(v)-\int_{\Omega} f \cdot v-\int_{\partial \Omega_{N}} g \cdot v \in \mathbb{R}, \\
& \Psi: H \ni q \longmapsto \Psi(q):=\int_{\Gamma} \psi(q) \in \mathbb{R},
\end{aligned}
$$

and the linear operator

$$
B: V \ni v \longmapsto B v:=\left.v\right|_{\Gamma} \cdot n \in H .
$$

The unilateral contact problem with cohesive forces can be expressed in the abstract variational form

$$
\left\{\begin{array}{l}
\min _{v \in V} W(v)+\Psi(B v) \\
\text { subject to } B v \in H^{+}
\end{array}\right.
$$

where $H^{+}:=\{q \in H ; q \geq 0\}$.

Problem (1.4) is a constrained minimization problem. For solving numerically such a problem, the main techniques are penalty methods, feasible direction methods, linear programming methods, and Lagrangian methods. These techniques are thoroughly discussed in [4]. The main drawbacks of the first three methods can be summarized in this way: penalty methods generally yield ill-conditioned problems, feasible direction methods are often expensive due to the projection step, and linear programming methods are limited to linear constraints and quadratic objective functions. In contrast, Lagrangian methods are based on a reformulation of the constrained minimization problem. The new problem consists in seeking a saddle-point (or a stationary point) of a Lagrangian. This can be achieved efficiently by Uzawa algorithms or Newton methods. Uzawa algorithms generally feature good global convergence properties (in the sense that they do not need an initialization value close to the optimum), but their speed of convergence is only linear. Newton methods feature a quadratic speed of convergence, but this is achieved only locally (that is, if the initialization is close to the optimum). Furthermore, augmenting the Lagrangian offers some additional advantages. Whenever the objective function is actually convex, this augmentation improves the performance of the algorithms. In the nonconvex case, the ordinary Lagrangian formulation is not necessarily well-posed and the augmentation enables to recover well-posedness. More details on augmented Lagrangian methods can be found in [3,4].

In the present work, we analyze two augmented Lagrangian methods for the problem of unilateral contact with cohesive forces: a decomposition-coordination method and a nonsmooth Newton's method. These two methods are based on the same three-field augmented Lagrangian formulation. The decomposition-coordination method has been proposed by Fortin and Glowinski [13] as a general method for solving nonlinear problems. The idea is to solve separately the linear and nonlinear parts of the problem at each iteration. This method can be seen as an Uzawa-like algorithm. It is closely related to the so-called Latin method [23] and also to splitting operator methods. Such methods have been applied to various unilateral contact problems, as for instance in [6,15]. In the case of a convex functional split into two convex parts, the convergence of the algorithm has been proved in [13]. Furthermore, Newton's method is a standard method for solving nonlinear systems of equations and, as such, can be used to find a stationary point of the augmented Lagrangian. In the case of unilateral constraints, the resulting system is only piecewise continuously differentiable and Newton's method can be extended to this class of nonsmooth systems [28]. Newton's method for unilateral contact problems has been used for instance in $[1,22]$. In particular, it has been applied to the problem of unilateral contact with cohesive forces in [25].

This paper is organized as follows. In Section 2, we specify the mathematical structure of the original constrained minimization problem (1.4) and investigate its well-posedness. In particular, we establish an existence result where the lack of convexity is compensated by a compactness argument. In Section 3, we introduce the three-field augmented Lagrangian formulation and study its well-posedness, namely the existence of a local saddle-point of the augmented Lagrangian. This result is well-known in the convex case [11]. In the nonconvex case, a result is available only in a finite-dimensional setting [3]. Here, we extend this latter approach 
to the (infinite-dimensional) problem of unilateral contact with cohesive forces, assuming the surjectivity of the operator $B$ defined by (1.3) and using a compactness argument in the (closure of the) cone of feasible directions. Sections 2 and 3 are set in a general framework encompassing the particular case of unilateral contact problems with cohesive forces. In Section 4, we analyze mixed finite element approximations of the augmented Lagrangian formulation of unilateral contact problems with cohesive forces. Since a nonlinear problem needs to be solved for the normal displacement on $\Gamma$, it is convenient to use a collocation method. In the same way, numerical integration can be employed to build the Jacobian matrix in Newton's method. A key point is the use of discontinuous finite element spaces leading to a collocation method, while ensuring an inf-sup condition which is the discrete counterpart of the surjectivity of the operator $B$. The resulting mixed finite element approximation is nonconforming. Numerous works have been devoted to the error analysis of mixed formulations for unilateral contact problems, especially for two-field formulations (bulk displacement-displacement on $\Gamma$ or bulk displacement-normal stress on $\Gamma$ ). To our knowledge, the only work dealing with the three-field augmented Lagrangian formulation is [7] in a conforming and consistent case. Here, we prove a priori error estimates in the present nonconforming setting for various finite element spaces under the simplifying assumption that the cohesive forces are mild enough. In Section 5, we describe the algorithms. We prove the convergence of the decomposition-coordination method in the particular case of a convex functional split into a convex part and a nonconvex part. Finally, numerical simulations illustrating the theoretical results are presented in Section 6 .

\section{WeLL-POSEDNESS OF THE CONTINUOUS PROBLEM}

The main result of this section is the existence of a minimizer for problem (1.4). The lack of convexity is compensated by a compactness argument. We also specify a sufficient condition for uniqueness based on $\alpha$-convexity and give some hints on the regularity of the solution.

We make the following assumptions on the mathematical structure of problem (1.4).

(H1) $V$ and $H$ are Hilbert spaces and $B \in \mathcal{L}(V, H)$ (the continuity constant is denoted by $c_{B}$ ).

(H2) $W$ is $\alpha$-convex on $V$ (the $\alpha$-convexity constant is denoted by $\alpha_{W}$ ).

(H3) $H^{+}$is a nonempty closed convex subset of $H$.

(H4) There is a Hilbert space $M \equiv M^{\prime}$ with scalar product $(\cdot, \cdot)_{M}$ such that $H \hookrightarrow M$ with compact imbedding (the continuity constant of the imbedding is denoted by $c_{M}$ ) and $\Psi: M \rightarrow \mathbb{R}$ is bounded and continuous.

(H5) $W$ and $\Psi$ are continuously differentiable on $V$ and $M$ respectively, and $\Psi^{\prime}$ is Lipschitz continuous on $M$ (the Lipschitz constant of $\Psi^{\prime}$ is denoted by $k_{\Psi^{\prime}}$ ).

Let $V^{+}:=\left\{v \in V ; B v \in H^{+}\right\}$, observe that $V^{+}$is a closed convex subset of $V$, and define the functional

$$
J: V \ni v \longmapsto J(v):=W(v)+\Psi(B v) \in \mathbb{R} .
$$

Problem (1.4) can be rewritten as

$$
\min _{v \in V^{+}} J(v) .
$$

Theorem 2.1. Assume (H1)-(H4). Then, there exists a solution to problem (1.4).

Proof. Let $\left(v_{n}\right)_{n \in \mathbb{N}}$ be a minimizing sequence of $J$ in $V^{+}$. Since the functional $J$ is coercive ( $W$ is $\alpha$-convex and $\Psi$ is bounded), the sequence $\left(v_{n}\right)_{n \in \mathbb{N}}$ is bounded in $V$. Hence, we can extract a subsequence, still denoted by $\left(v_{n}\right)_{n \in \mathbb{N}}$, which converges weakly to $v_{\infty}$ in $V$. The limit $v_{\infty}$ belongs to $V^{+}$since a strongly closed convex set is weakly closed. Moreover, owing to the continuity of $B$ from $V$ to $H$ and the compactness of the imbedding $H \hookrightarrow M$, the sequence $\left(B v_{n}\right)_{n \in \mathbb{N}}$ strongly converges to $B v_{\infty}$ in $M$. Using the continuity of $\Psi$ on $M$, we conclude that $\lim _{n \rightarrow \infty} \Psi\left(B v_{n}\right)=\Psi\left(B v_{\infty}\right)$. Furthermore, since the functional $W$ is convex and continuous on $V, \liminf _{n \rightarrow \infty} W\left(v_{n}\right) \geq W\left(v_{\infty}\right)$. Thus, $v_{\infty} \in V^{+}$is a global minimizer of $J$ in $V^{+}$.

Proposition 2.2. Assume (H1)-(H5). Then, $J$ is differentiable on $V$ so that a solution $u$ to (1.4) satisfies

$$
\left\langle J^{\prime}(u), v-u\right\rangle_{V^{\prime}, V} \geq 0, \quad \forall v \in V^{+} .
$$


Furthermore, if

$$
\alpha_{W}-k_{\Psi^{\prime}} c_{M}^{2} c_{B}^{2}>0
$$

then $J$ is $\alpha$-convex on $V$ and the solution to (1.4) is unique.

Proof. The first statement is evident. Concerning the second one, observe that for all $(v, w) \in V \times V$,

$$
\begin{aligned}
\left\langle J^{\prime}(v)-J^{\prime}(w), v-w\right\rangle_{V^{\prime}, V} & \geq\left\langle W^{\prime}(v)-W^{\prime}(w), v-w\right\rangle_{V^{\prime}, V}+\left(\Psi^{\prime}(B v)-\Psi^{\prime}(B w), B v-B w\right)_{M} \\
& \geq \alpha_{W}\|v-w\|_{V}^{2}-k_{\psi^{\prime}}\|B v-B w\|_{M}^{2} \\
& \geq \alpha_{W}\|v-w\|_{V}^{2}-k_{\psi^{\prime}} c_{M}^{2}\|B v-B w\|_{H}^{2} \\
& \geq\left(\alpha_{W}-k_{\Psi^{\prime}} c_{M}^{2} c_{B}^{2}\right)\|v-w\|_{V}^{2}
\end{aligned}
$$

which proves the $\alpha$-convexity of $J$ under the condition (2.4), and hence the uniqueness of the solution.

Remark 2.3. Relation (2.3) links problem (1.4) to the theory of variational inequalities. When $J$ is convex, the operator $J^{\prime}$ is monotone. In the general case, the proof of Theorem 2.1 shows that $J^{\prime}$ is pseudo-monotone.

We now verify that the unilateral contact problem with cohesive forces defined in the introduction fits the above abstract framework. Recalling the definitions (1.1)-(1.3) of $W, \Psi$, and $B$, we also set

$$
V:=\left\{v \in H^{1}(\Omega)^{d} ;\left.v\right|_{\partial \Omega_{D}}=0\right\}, \quad H:=H_{00}^{\frac{1}{2}}\left(\Gamma, \partial \Gamma_{0}\right), \quad M:=\mathrm{L}^{2}(\Gamma),
$$

where $\partial \Gamma_{0}:=\overline{\partial \Omega_{D}} \cap \bar{\Gamma}$ (see Fig. 1). The space $H_{00}^{\frac{1}{2}}\left(\Gamma, \partial \Gamma_{0}\right)$ is the space of functions of $H^{\frac{1}{2}}(\Gamma)$ that are zero in a certain sense on $\partial \Gamma_{0}$. It can be built by interpolation between $L^{2}(\Gamma)$ and $H_{0}^{1}\left(\Gamma, \partial \Gamma_{0}\right)$; see [24] for more details. Furthermore, $H^{+}:=\{q \in H ; q \geq 0$ a.e. in $\Gamma\}$ and observe that with the above notation,

$$
\Psi(q)=(\psi(q), 1)_{M}
$$

Finally, for further use, we set $M^{+}=\{q \in M ; q \geq 0$ a.e. in $\Gamma\}$.

Proposition 2.4. Assumptions (H1)-(H3) hold. If $\psi$ is continuous and bounded on $\mathbb{R}$, assumption (H4) holds. If $\psi^{\prime}$ is Lipschitz continuous on $\mathbb{R}$ with Lipschitz constant $k_{\psi^{\prime}}$, assumption (H5) holds with $k_{\Psi^{\prime}}=k_{\psi^{\prime}}$.

Proof. Assumption (H1) holds by construction. Assumption (H2) is a direct consequence of Korn's first inequality [8]. Assumption (H3) is readily verified. Concerning assumptions (H4) and (H5), we first observe that, by construction, $H_{00}^{\frac{1}{2}}\left(\Gamma, \partial \Gamma_{0}\right)$ is compactly imbedded in $L^{2}(\Gamma)$. Furthermore, to prove the regularity of $\Psi$, we use a basic result of nonlinear analysis [10]; see Lemma 2.5 below. Using this lemma with $\phi=\psi, p=2$, and $q=1$ along with the boundedness of $\psi$ to verify condition (2.6), we infer that $\mathcal{S}_{\psi}$ is continuous from $L^{2}(\Gamma)$ into $L^{1}(\Gamma)$. Since for all $q \in L^{2}(\Gamma), \Psi(q)=\left(\mathcal{S}_{\psi}(q), 1\right)_{M}$, the operator $\Psi$ is continuous on $M$. Moreover, since for all $q, r \in L^{2}(\Gamma)$,

$$
\begin{aligned}
\Psi(q+r)-\Psi(q)-\left(\mathcal{S}_{\psi^{\prime}}(q), r\right)_{M} & =\int_{\Gamma}\left(\int_{0}^{1}\left(\psi^{\prime}(q(x)+t r(x))-\psi^{\prime}(q(x))\right) \mathrm{d} t\right) r(x) \mathrm{d} x \\
& \leq \frac{1}{2} k_{\psi^{\prime}} \int_{\Gamma}|r(x)|^{2} \mathrm{~d} x
\end{aligned}
$$

owing to the Lipschitz continuity of $\psi^{\prime}, \Psi$ is differentiable on $M$ with $\left(\Psi^{\prime}(q), r\right)_{M}=\left(\mathcal{S}_{\psi^{\prime}}(q), r\right)_{M}$. Using Lemma 2.5 with $\phi=\psi^{\prime}$ and $p=q=2$ along with the Lipschitz continuity of $\psi^{\prime}$ readily shows that $\Psi^{\prime}$ is Lipschitz continuous on $M$ with Lipschitz constant $k_{\psi^{\prime}}$. Finally, the differentiability of $W$ is obvious. 
Lemma 2.5. Let $\phi: \mathbb{R} \rightarrow \mathbb{R}$ be a continuous function. Consider a measurable function $q: \Gamma \subset \mathbb{R}^{n} \rightarrow \mathbb{R}$. The superposition operator (or Nemitsky operator) $\mathcal{S}_{\phi}$ maps $q$ to $\phi \circ q$. If $q$ and $r$ are measurable functions that coincide almost everywhere on $\Gamma$, then $\mathcal{S}_{\phi}(q)$ and $\mathcal{S}_{\phi}(r)$ are measurable functions that coincide almost everywhere on $\Gamma$. Moreover, if $\phi$ satisfies the growth condition,

$$
\exists a, b \in \mathbb{R}, \quad \forall x \in \mathbb{R}, \quad|\phi(x)| \leq a+b|x|^{p / q},
$$

then the superposition operator maps $L^{p}(\Gamma)$ into $L^{q}(\Gamma)$ and is strongly continuous $(p, q \in[1 ;+\infty[)$.

Remark 2.6. The $\alpha$-convexity condition (2.4) can be interpreted in terms of the problem parameters. The constant $\alpha_{W}$ is proportional to the Young modulus of the material. The constant $k_{\psi^{\prime}}$ is larger when the cohesive forces decrease fast. By a scaling argument, it can be seen that $c_{M} c_{B}$ decreases to zero with the $(d-1)$-dimensional measure $|\Gamma|$. Thus, condition (2.4) is more likely to be met when the Young modulus is large, the cohesive force decreases slowly, or $|\Gamma|$ is small.

A detailed study of the regularity of the solution to the minimization problem (1.4) is beyond the scope of the present work. However, let us mention some results in particular cases. For a unilateral contact problem without cohesive forces under body forces in $L^{2}(\Omega)^{d}$, the displacement is in $H_{\mathrm{loc}}^{2}(\Omega \cup \Gamma)^{d}[21]$. Furthermore, for a scalar elliptic problem in $2 \mathrm{D}$ with unilateral contact and homogeneous Dirichlet condition, the regularity of the solution has been studied near the junction between these boundary conditions [27]. Under body forces in $L^{2}(\Omega)$ and for a smooth junction, the solution is in $H^{\frac{3}{2}}(\Omega)$. For an angular junction (of internal angle $\omega$ ), the solution is in $H^{2}(\Omega)$ if $\omega \leq \pi / 2$, and in $H^{1+\frac{\pi}{2 \omega}}(\Omega)$ otherwise. With a sufficiently smooth cohesive law, it seems reasonable to expect at least the same kind of regularity. Furthermore, cohesive zone models have been designed to avoid unphysical infinite stresses at the crack tip. Yet, the question whether an appropriate choice of cohesive interface and law can lead to more regular solutions is still mathematically open.

\section{A three-Field AUgmented Lagrangian Formulation}

We introduce a new unknown $q$ representing the normal displacement on $\Gamma$. The decomposed problem is

$$
\left\{\begin{array}{l}
\min _{(v, q) \in V \times H^{+}} W(v)+\Psi(q) \\
\text { subject to } B v=q .
\end{array}\right.
$$

The decomposed problem (3.1), which is obviously equivalent to the initial minimization problem (1.4), is a minimization problem under a linear equality constraint. We treat this constraint by an augmented Lagrangian method. Introduce the space $Y:=V \times H$ (equipped with its natural norm) and the convex set $K:=V \times H^{+}$. Define

$$
\begin{aligned}
& J_{0}: Y \ni y:=(v, q) \longmapsto J_{0}(y):=W(v)+\Psi(q) \in \mathbb{R}, \\
& \tilde{B}: Y \ni y:=(v, q) \longmapsto \tilde{B} y:=B v-q \in H,
\end{aligned}
$$

so that (3.1) amounts to

$$
\min _{y \in K \cap \operatorname{ker} \tilde{B}} J_{0}(y)
$$

The augmented Lagrangian associated with the decomposed problem is

$$
L_{r}: Y \times H^{\prime} \ni(y, \lambda) \longmapsto L_{r}(y, \lambda):=J_{0}(y)+\langle\lambda, \tilde{B} y\rangle_{H^{\prime}, H}+\frac{r}{2}\|\tilde{B} y\|_{M}^{2} \in \mathbb{R},
$$

where $r$ is an arbitrary non-negative constant. For $y \in Y$, set

$$
J_{r}(y):=J_{0}(y)+\frac{r}{2}\|\tilde{B} y\|_{M}^{2} .
$$


A couple $(x, \theta) \in K \times H^{\prime}$ is said to be a local saddle-point of the augmented Lagrangian if it satisfies

$$
\forall \lambda \in H^{\prime}, \quad L_{r}(x, \lambda) \leq L_{r}(x, \theta) \leq L_{r}(y, \theta), \quad \forall y \in U,
$$

where $U \subset K$ is a neighborhood of $x$. The introduction of the augmented Lagrangian is motivated by the following proposition whose proof is straightforward.

Proposition 3.1. If $(x, \theta) \in K \times H^{\prime}$ is a local saddle-point of the augmented Lagrangian, then $x$ is a local minimizer of the decomposed problem (3.1).

The converse of this statement is more difficult to establish. We first prove, under the key assumption that $B$ is surjective from $V$ to $H$, that if $x \in K \cap \operatorname{ker} \tilde{B}$ is a local minimizer of $J_{0}$, there is (a unique) $\theta \in H^{\prime}$ such that $(x, \theta)$ is a stationary point of the augmented Lagrangian $L_{r}$. Then, we prove, under an additional assumption, that such a stationary point is a local saddle-point of $L_{r}$. A couple $(x, \theta) \in K \times H^{\prime}$ is said to be a stationary point of $L_{r}$ if it satisfies

$$
\begin{array}{ll}
\left\langle\partial_{y} L_{r}(x, \theta), y-x\right\rangle_{Y^{\prime}, Y} \equiv\left\langle J_{r}^{\prime}(x), y-x\right\rangle_{Y^{\prime}, Y}+\langle\theta, \tilde{B}(y-x)\rangle_{H^{\prime}, H} \geq 0, & \forall y \in K, \\
\left\langle\partial_{\lambda} L_{r}(x, \theta), \lambda\right\rangle_{H, H^{\prime}} \equiv\langle\lambda, \tilde{B} x\rangle_{H^{\prime}, H}=0, & \forall \lambda \in H^{\prime} .
\end{array}
$$

Observe that being a stationary point of the augmented Lagrangian is a property independent of $r$ since (3.7) implies $\tilde{B} x=0$ so that $J_{r}^{\prime}(x)=J_{0}^{\prime}(x)$. Notice also that (3.6) can be rewritten for $x:=(u, p)$ as

$$
\begin{array}{ll}
\left\langle W^{\prime}(u), v\right\rangle_{V^{\prime}, V}+\langle\theta, B v\rangle_{H^{\prime}, H}=0, & \forall v \in V, \\
\left(\psi^{\prime}(p), q-p\right)_{M}-\langle\theta, q-p\rangle_{H^{\prime}, H} \geq 0, & \forall q \in H^{+} .
\end{array}
$$

Proposition 3.2. Let $x \in K \cap$ ker $\tilde{B}$ be a local minimizer of the decomposed problem (3.1). If $B$ is surjective from $V$ to $H$, there exists a unique $\theta \in H^{\prime}$ such that $(x, \theta)$ is a stationary point of the augmented Lagrangian.

Proof. Let $x \in K \cap$ ker $\tilde{B}$ be a local minimizer of the decomposed problem. Then, $\tilde{B} x=0$ and (3.7) obviously holds. Let us now prove (3.6). For all $r \geq 0, x$ minimizes $J_{r}$ over $K \cap$ ker $\tilde{B}$ and hence it satisfies

$$
\left\langle J_{r}^{\prime}(x), y-x\right\rangle_{Y^{\prime}, Y} \geq 0, \quad \forall y \in K \cap \operatorname{ker} \tilde{B} .
$$

For all $v \in \operatorname{ker} B, y:=x+(v, 0)$ belongs to $K \cap \operatorname{ker} \tilde{B}$ so that $\left\langle J_{r}^{\prime}(x),(v, 0)\right\rangle_{Y^{\prime}, Y}=0$. Since $B$ is surjective, $(\operatorname{ker} B)^{\perp}=\operatorname{im~} B^{*}$ by the closed range theorem. As a consequence, there exists $\theta \in H^{\prime}$ such that

$$
\left\langle J_{r}^{\prime}(x),(v, 0)\right\rangle_{Y^{\prime}, Y}+\langle\theta, B v\rangle_{H^{\prime}, H}=0, \quad \forall v \in V .
$$

Since $J_{r}^{\prime}(x)=J_{0}^{\prime}(x), \theta$ does not depend on $r$. Now let $y:=(v, q) \in K$ and let $w \in V$ be such that $B w=q$. Then,

$$
\begin{aligned}
\left\langle J_{r}^{\prime}(x), y-x\right\rangle_{Y^{\prime}, Y}+\langle\theta, \tilde{B}(y-x)\rangle_{H^{\prime}, H} & =\left\langle J_{r}^{\prime}(x), y-x\right\rangle_{Y^{\prime}, Y}+\langle\theta, B(v-w)\rangle_{H^{\prime}, H} \\
& =\left\langle J_{r}^{\prime}(x),(w, q)-x\right\rangle_{Y^{\prime}, Y} \geq 0,
\end{aligned}
$$

since $(w, q)$ is by construction in $K \cap \operatorname{ker} \tilde{B}$. Hence, (3.6) also holds. Finally, the relation $\left\langle J_{r}^{\prime}(x),(v, 0)\right\rangle_{Y^{\prime}, Y}+$ $\langle\theta, B v\rangle_{H^{\prime}, H}=0$ for all $v \in V$ and the surjectivity of $B$ from $V$ to $H$ imply that $\theta$ is unique.

Remark 3.3. In the context of unilateral contact problems, the Lagrange multiplier $\theta$ can be interpreted as the normal stress on $\Gamma$, namely $\theta=\sigma(u)_{\Gamma}$ where $x:=\left(u, u_{\Gamma}\right)$. This results from the relation (3.6).

Remark 3.4. A more general existence result for mixed linear variational inequalities can be found in [29]. 
We now examine whether a stationary point of the augmented Lagrangian is a local saddle-point. The cone of feasible directions at the point $x:=(u, p) \in K$ can be defined as $\left(V \times C_{+}(x)\right) \cap$ ker $\tilde{B}$ where

$$
C_{+}(x):=\left\{d \in H ; \exists \alpha>0, p+\alpha d \in H^{+}\right\} .
$$

Proposition 3.5. Assume that $W$ and $\Psi$ are of class $\mathrm{C}^{2}$. Let $(x, \theta) \in K \times H^{\prime}$ be a stationary point of the augmented Lagrangian. Assume that $(x, \theta)$ satisfies the following second-order condition (indices on brackets are dropped for second-order derivatives)

$$
\left\langle J_{0}^{\prime \prime}(x),(d, d)\right\rangle>0, \quad \forall d \in\left(V \times \overline{C_{+}(x)}\right) \cap \operatorname{ker} \tilde{B} \backslash\{0\} .
$$

Then, there exists $r_{0} \geq 0$ such that $(x, \theta)$ is a local saddle-point of the augmented Lagrangian $L_{r_{0}}$. Furthermore, for all $r \geq r_{0},(x, \theta)$ is a local saddle-point of the augmented Lagrangian $L_{r}$.

Proof. The left inequality in (3.5) is obvious for all $r \geq 0$. If the right inequality holds for $r_{0} \geq 0$, then it holds also for $r \geq r_{0}$. Now we shall prove by contradiction that there exist $r_{0} \geq 0$ and a neighborhood $U$ of $x$ such that $L_{r}(x, \theta) \leq L_{r}(y, \theta), \forall y \in U, \forall r \geq r_{0}$. Suppose there exists a sequence of positive reals $\left(r_{k}\right)_{k \in \mathbb{N}}$ tending to infinity and a sequence $\left(x_{k}\right)_{k \in \mathbb{N}}$ of elements of $K$ tending to $x$ such that

$$
L_{r_{k}}\left(x_{k}, \theta\right) \leq L_{r_{k}}(x, \theta)
$$

Consider the sequence $\left(e_{k}\right)_{k \in \mathbb{N}}$ such that $e_{k}:=\left(e_{k}^{v}, e_{k}^{q}\right):=\varrho_{k}^{-1}\left(x_{k}-x\right)$ where $\varrho_{k}:=\left\|x_{k}-x\right\|_{Y}$. Since this sequence is bounded in $Y$, there exists a subsequence, still denoted by $\left(e_{k}\right)_{k \in \mathbb{N}}$, weakly converging to $e:=\left(e^{v}, e^{q}\right)$ in $Y$. To obtain a contradiction, we shall now prove that $e \in\left(V \times \overline{C_{+}(x)}\right) \cap \operatorname{ker} \tilde{B}$ and that $\left\langle J_{0}^{\prime \prime}(x),(e, e)\right\rangle \leq 0$. A second-order Taylor expansion of $L_{0}(\cdot, \theta)$ at $x$ in the $Y$-norm yields

$$
L_{0}\left(x_{k}, \theta\right)=L_{0}(x, \theta)+\left\langle\partial_{y} L_{0}(x, \theta), x_{k}-x\right\rangle_{Y^{\prime}, Y}+\frac{1}{2}\left\langle J_{0}^{\prime \prime}(x),\left(x_{k}-x, x_{k}-x\right)\right\rangle+o\left(\varrho_{k}^{2}\right) .
$$

Since $x_{k}=x+\varrho_{k} e+\varrho_{k}\left(e_{k}-e\right)$,

$$
\begin{aligned}
L_{0}\left(x_{k}, \theta\right)= & L_{0}(x, \theta)+\left\langle\partial_{y} L_{0}(x, \theta), x_{k}-x\right\rangle_{Y^{\prime}, Y}+\alpha_{k}^{2}\left\langle J_{0}^{\prime \prime}(x),\left(e_{k}-e, e\right)\right\rangle \\
& +\frac{\alpha_{k}^{2}}{2}\left\langle J_{0}^{\prime \prime}(x),(e, e)\right\rangle+\frac{\alpha_{k}^{2}}{2}\left\langle J_{0}^{\prime \prime}(x),\left(e_{k}-e, e_{k}-e\right)\right\rangle+o\left(\varrho_{k}^{2}\right) .
\end{aligned}
$$

Since $(x, \theta)$ is a stationary point of the augmented Lagrangian, $\left\langle\partial_{y} L_{0}(x, \theta), x_{k}-x\right\rangle_{Y^{\prime}, Y} \geq 0$. Now observe that $\tilde{B} x_{k}=\tilde{B} x+\varrho_{k} \tilde{B} e_{k}=\varrho_{k} \tilde{B} e_{k}$. Hence, substituting (3.13) into (3.12), it is inferred that

$$
\alpha_{k}^{2}\left\langle J_{0}^{\prime \prime}(x),\left(e_{k}-e, e\right)\right\rangle+\frac{\alpha_{k}^{2}}{2}\left\langle J_{0}^{\prime \prime}(x),(e, e)\right\rangle+\frac{\alpha_{k}^{2}}{2}\left\langle J_{0}^{\prime \prime}(x),\left(e_{k}-e, e_{k}-e\right)\right\rangle+\frac{r_{k}}{2} \varrho_{k}^{2}\left\|\tilde{B} e_{k}\right\|_{M}^{2}+o\left(\varrho_{k}^{2}\right) \leq 0 .
$$

Since the sequence $\left(e_{k}\right)_{k \in \mathbb{N}}$ converges weakly to $e$ in $Y,\left\langle J_{0}^{\prime \prime}(x),\left(e_{k}-e, e\right)\right\rangle$ tends to 0 . By convexity $\left\langle W^{\prime \prime}(x),\left(e_{k}^{v}-\right.\right.$ $\left.\left.e^{v}, e_{k}^{v}-e^{v}\right)\right\rangle \geq 0$ and by compactness, $e_{k}^{q}$ tends to $e^{q}$ in $M$ so that $\left\langle\Psi^{\prime \prime}(x),\left(e_{k}^{q}-e^{q}, e_{k}^{q}-e^{q}\right)\right\rangle$ tends to 0 . Hence, $\liminf _{k}\left\langle J_{0}^{\prime \prime}(x),\left(e_{k}-e, e_{k}-e\right)\right\rangle \geq 0$. By compactness, the sequence $\left(\tilde{B} e_{k}\right)_{k \in \mathbb{N}}$ converges strongly to $\tilde{B} e$ in $M$. Dividing (3.14) by $\varrho_{k}^{2} r_{k}$ and passing to the limit, we obtain $\|\tilde{B} e\|_{M}^{2} \leq 0$ and thus $e \in \operatorname{ker} \tilde{B}$. Moreover, since $x_{k}=x+\varrho_{k} e_{k}$, it is clear that for all $k \geq 0, e_{k}^{q} \in C_{+}(x)$. Observing that $C_{+}(x)$ is convex, it is inferred that $e^{q} \in \overline{C_{+}(x)}$. Hence, $e \in\left(V \times \overline{C_{+}(x)}\right) \cap$ ker $\tilde{B}$; furthermore, by construction, $e \neq 0$. Finally, dividing (3.14) by $\varrho_{k}^{2}$, dropping the positive terms, and passing to the limit leads to $\left\langle J_{0}^{\prime \prime}(x),(e, e)\right\rangle \leq 0$. 


\section{Approximation By MiXed Finite ElEMENTS}

In this section, we discretize the augmented Lagrangian formulation of unilateral contact problems with cohesive forces by a Galerkin method with finite element spaces. The augmented Lagrangian formulation is a three-field formulation: the bulk displacement, the normal displacement on $\Gamma$, and the Lagrange multiplier (which can be interpreted as the normal stress on $\Gamma$ ). The two key ideas in the design of the mixed finite element approximation are the following. Firstly, we want to solve the nonlinear part of the problem concerning the normal displacement on $\Gamma$ by a collocation method. This leads to the use of discontinuous finite element spaces spanned by nodal basis functions for approximating this quantity. Secondly, a surjectivity condition in the form of a discrete inf-sup condition must be satisfied, linking the discrete spaces for the bulk displacement and the Lagrange multiplier. In the sequel, we refer to a $3 \mathrm{D} / 2 \mathrm{D}$ setting when $\Omega$ is $3 \mathrm{D}$ and $\Gamma$ is $2 \mathrm{D}$, and to a $2 \mathrm{D} / 1 \mathrm{D}$ setting when $\Omega$ is $2 \mathrm{D}$ and $\Gamma$ is $1 \mathrm{D}$. Moreover, $A \lesssim B$ means the inequality $A \leq c B$ with a positive constant $c$ independent of the mesh-size. Henceforth, the minimal regularity requirement for $\psi$ is that $\psi^{\prime}$ is Lipschitz continuous.

\subsection{The discrete setting}

Let $\left\{\mathcal{T}_{h}\right\}_{h>0}$ be a shape-regular family of affine meshes covering exactly $\Omega$, where the parameter $h$ stands for the maximum size of the elements in $\mathcal{T}_{h}$. Without loss of generality, we assume $h \leq 1$. Let $\mathcal{F}_{h}$ collect the mesh faces located on $\Gamma$. To alleviate technicalities, the mesh family $\left\{\mathcal{F}_{h}\right\}_{h>0}$ is assumed to be quasi-uniform on $\Gamma$, but this assumption can be relaxed. Let $V_{h}, M_{h}$, and $\Lambda_{h}$ respectively denote the finite element approximation spaces for the bulk displacement, the normal displacement on $\Gamma$, and the Lagrange multiplier. Henceforth, we assume that

$$
V_{h} \subset V, \quad \text { and } \quad \Lambda_{h} \subset M_{h} \subset M .
$$

Thus, the approximation is conforming for the bulk displacement and the Lagrange multiplier, but not for the normal displacement on $\Gamma$ since in general $M_{h} \not \subset H$. In fact, motivated by the use of a collocation method, we will choose $M_{h}$ as a discontinuous finite element space spanned by nodal basis functions; see Remark 4.6 below for further insight. Let $\Pi_{\Lambda_{h}}$ denote the $L^{2}$-orthogonal projection from $M$ onto $\Lambda_{h}$ and define the operator

$$
B_{h}: V \ni v \longmapsto B_{h} v:=\Pi_{\Lambda_{h}} B v \in \Lambda_{h} .
$$

The choice for the spaces $V_{h}$ and $\Lambda_{h}$ is linked by the following discrete inf-sup condition

$$
\exists \beta_{h}>0, \quad \forall \lambda_{h} \in \Lambda_{h}, \quad \beta_{h} h^{1 / 2}\left\|\lambda_{h}\right\|_{M} \leq \sup _{v_{h} \in V_{h}} \frac{\left(B_{h} v_{h}, \lambda_{h}\right)_{M}}{\left\|v_{h}\right\|_{V}} .
$$

This means that the restriction of the operator $B_{h}$ to $V_{h}$ is surjective onto $\Lambda_{h}$. Henceforth, we assume that this condition holds.

Remark 4.1. The scaling factor $h^{1 / 2}$ has been introduced since the natural norm for $\lambda_{h}$ is the $H^{-\frac{1}{2}}$-norm.

Consider the following finite element spaces

$$
\begin{aligned}
\mathcal{P}_{\mathrm{c}}^{k}\left(\mathcal{T}_{h}\right) & =\left\{v_{h} \in \mathrm{C}^{0}(\Omega) ; \forall T \in \mathcal{T}_{h},\left.v_{h}\right|_{T} \in \mathbb{P}_{k}\right\}, \\
\mathcal{P}_{\mathrm{d}}^{k}\left(\mathcal{F}_{h}\right) & =\left\{q_{h} \in \mathrm{L}^{2}(\Gamma) ; \forall F \in \mathcal{F}_{h},\left.q_{h}\right|_{F} \in \mathbb{P}_{k}\right\}, \quad \mathcal{P}_{\mathrm{c}}^{k}\left(\mathcal{F}_{h}\right)=\mathcal{P}_{\mathrm{d}}^{k}\left(\mathcal{F}_{h}\right) \cap \mathrm{C}^{0}(\Gamma),
\end{aligned}
$$

where for an integer $k, \mathbb{P}_{k}$ denotes the space of polynomials with total degree $\leq k$. We are interested in analyzing the following situations

$$
\begin{aligned}
& M_{h}=\mathcal{P}_{\mathrm{d}}^{0}\left(\mathcal{F}_{h}\right), \quad \Lambda_{h}=M_{h}, \quad V_{h} \supset \mathcal{P}_{\mathrm{c}}^{1}\left(\mathcal{T}_{h}\right)^{d}, \\
& M_{h}=\mathcal{P}_{\mathrm{d}}^{1}\left(\mathcal{F}_{h}\right), \quad \Lambda_{h}=M_{h}, \quad V_{h} \supset \mathcal{P}_{\mathrm{c}}^{2}\left(\mathcal{T}_{h}\right)^{d}, \\
& M_{h}=\mathcal{P}_{\mathrm{d}}^{1}\left(\mathcal{F}_{h}\right), \quad \Lambda_{h}=\mathcal{P}_{\mathrm{c}}^{1}\left(\mathcal{F}_{h}\right), \quad V_{h}=\mathcal{P}_{\mathrm{c}}^{2}\left(\mathcal{T}_{h}\right)^{d} .
\end{aligned}
$$


In (4.6) and (4.7), the most robust choice is to take for $V_{h}$, respectively, the continuous first-order and secondorder finite element spaces augmented with suitable face bubbles on $\Gamma$, leading to an inf-sup constant $\beta_{h}$ in (4.3) independent of $h$ in both $2 \mathrm{D} / 1 \mathrm{D}$ and $3 \mathrm{D} / 2 \mathrm{D}$ settings; see $[2,17]$. In $2 \mathrm{D} / 1 \mathrm{D}$ whenever at least one of the endpoints of $\Gamma$ is free, it is also possible to take $V_{h}=\mathcal{P}_{\mathrm{c}}^{1}\left(\mathcal{T}_{h}\right)^{d}$ in (4.6) or $V_{h}=\mathcal{P}_{\mathrm{c}}^{2}\left(\mathcal{T}_{h}\right)^{d}$ in (4.7); then, the discrete inf-sup condition (4.3) still holds, but the constant $\beta_{h}$ is of order $h$. The choice (4.8) has been introduced in [25] and differs from the two previous choices in the fact that $\Lambda_{h} \neq M_{h}$. The idea is to avoid the use of face bubbles on $\Gamma$ by simply taking $V_{h}=\mathcal{P}_{\mathrm{c}}^{2}\left(\mathcal{T}_{h}\right)^{d}$, to ensure a robust discrete inf-sup condition (with $\beta_{h}$ independent of $h$ ) by restricting $\Lambda_{h}$ to $\mathcal{P}_{\mathrm{c}}^{1}\left(\mathcal{F}_{h}\right)$, and to keep $M_{h}$ as a discontinuous finite element space to be able to use a collocation method.

In all cases resulting from (4.6)-(4.8), there holds $M_{h}=\mathcal{P}_{\mathrm{d}}^{k}\left(\mathcal{F}_{h}\right)$ with $k \in\{0,1\}$, and it is readily verified that there is a family of nodes $\left(\xi_{i}^{F}\right)_{1 \leq i \leq n_{q}, F \in \mathcal{F}_{h}}$ such that

- the associated shape functions form a basis of $M_{h}$ (in 2D/1D, $n_{q}=k+1$ and the usual Gauss nodes are used; in $3 \mathrm{D} / 2 \mathrm{D}$, if $k=0, n_{q}=1$ and the barycenter of each $F \in \mathcal{F}_{h}$ is used, while if $k=1, n_{q}=3$ and the midpoints of the three edges of each $F \in \mathcal{F}_{h}$ are used);

- there are positive weights $\left(\omega_{i}^{F}\right)_{1 \leq i \leq n_{q}, F \in \mathcal{F}_{h}}$ such that for all $q_{h}, r_{h} \in M_{h}$,

$$
\left(q_{h}, r_{h}\right)_{M}=\sum_{F \in \mathcal{F}_{h}} \sum_{i=1}^{n_{q}} \omega_{i}^{F} q_{h}\left(\xi_{i}^{F}\right) r_{h}\left(\xi_{i}^{F}\right)
$$

In other words, on all $F \in \mathcal{F}_{h}$, the quadrature with nodes $\left(\xi_{i}^{F}\right)_{1 \leq i \leq n_{q}}$ and weights $\left(\omega_{i}^{F}\right)_{1 \leq i \leq n_{q}}$ is at least of degree $2 k$. For further use, it is convenient to define the bilinear form

$$
\mathrm{C}^{0}\left(\mathcal{F}_{h}\right) \times \mathrm{C}^{0}\left(\mathcal{F}_{h}\right) \ni\left(q_{h}, r_{h}\right) \longmapsto\left(q_{h}, r_{h}\right)_{M_{h}}:=\sum_{F \in \mathcal{F}_{h}} \sum_{i=1}^{n_{q}} \omega_{i}^{F} q_{h}\left(\xi_{i}^{F}\right) r_{h}\left(\xi_{i}^{F}\right) \in \mathbb{R}
$$

where $\mathrm{C}^{0}\left(\mathcal{F}_{h}\right)$ denotes the space of functions whose restriction to every $F \in \mathcal{F}_{h}$ is continuous.

\subsection{The discrete augmented Lagrangian formulation}

Set $Y_{h}=V_{h} \times M_{h}$ and $K_{h}=V_{h} \times M_{h}^{+}$where

$$
M_{h}^{+}:=\left\{q_{h} \in M_{h} ; \forall F \in \mathcal{F}_{h}, \forall 1 \leq i \leq n_{q}, q_{h}\left(\xi_{i}^{F}\right) \geq 0\right\} .
$$

Observe that $M_{h}^{+} \subset M^{+}$if $k=0$ (that is, functions in $M_{h}^{+}$are indeed non-negative), whereas this is no longer the case if $k=1$, thereby introducing an additional source of nonconformity in the approximation. Let

$$
\tilde{B}_{h}: Y_{h} \ni y_{h}:=\left(v_{h}, q_{h}\right) \longmapsto \tilde{B}_{h} y_{h}:=\Pi_{\Lambda_{h}}\left(B v_{h}-q_{h}\right) \in \Lambda_{h} .
$$

Whenever $\Lambda_{h} \neq M_{h}$, we will also need the operator

$$
\tilde{B}_{h}^{\sharp}: Y_{h} \ni y_{h}:=\left(v_{h}, q_{h}\right) \longmapsto \tilde{B}_{h}^{\sharp} y_{h}:=\Pi_{M_{h}} B v_{h}-q_{h} \in M_{h},
$$

where $\Pi_{M_{h}}$ denotes the $L^{2}$-orthogonal projection from $M$ onto $M_{h}$. We define the discrete augmented Lagrangian as

$$
L_{r, h}: Y_{h} \times \Lambda_{h} \ni\left(y_{h}, \lambda_{h}\right) \longmapsto L_{r, h}\left(y_{h}, \lambda_{h}\right):=J_{0, h}\left(y_{h}\right)+\left(\lambda_{h}, \tilde{B}_{h} y_{h}\right)_{M}+\frac{r}{2}\left\|\tilde{B}_{h}^{\sharp} y_{h}\right\|_{M}^{2} \in \mathbb{R},
$$

where $r$ is a non-negative parameter. Here, for $y_{h}:=\left(v_{h}, q_{h}\right) \in Y_{h}$,

$$
J_{0, h}\left(y_{h}\right):=W\left(v_{h}\right)+\left(\psi\left(q_{h}\right), 1\right)_{M_{h}},
$$


that is, the energy associated with the cohesive forces is evaluated using a quadrature, and it is convenient to set

$$
J_{r, h}\left(y_{h}\right):=J_{0, h}\left(y_{h}\right)+\frac{r}{2}\left\|\tilde{B}_{h}^{\sharp} y_{h}\right\|_{M}^{2} .
$$

Observe that the penalty term in (4.14) and in (4.16) is stronger than the usual penalty term associated with the constraint $\tilde{B}_{h} y_{h}=0$ in $\Lambda_{h}$; indeed, owing to the fact that $\Lambda_{h} \subset M_{h}$, there holds

$$
\forall y_{h} \in Y_{h}, \quad\left\|\tilde{B}_{h} y_{h}\right\|_{M} \leq\left\|\tilde{B}_{h}^{\sharp} y_{h}\right\|_{M} .
$$

The discrete decomposed problem takes the following form

$$
\min _{y_{h} \in K_{h} \cap \operatorname{ker} \tilde{B}_{h}} J_{r, h}\left(y_{h}\right) .
$$

Proposition 4.2. There exists a solution to the discrete decomposed problem (4.18).

Proof. The functional $J_{r, h}$ is coercive and continuous, and the set $K_{h} \cap$ ker $\tilde{B}_{h}$ is nonempty and closed. In finite dimension, this suffices for the existence of a global minimizer.

We now investigate sufficient conditions for the functional $J_{r, h}$ to be $\alpha$-convex over $K_{h} \cap$ ker $\tilde{B}_{h}$ (and thus the solution of (4.18) to be unique). Since we are working in a nonconforming framework $\left(M_{h} \subset M\right.$, but $\left.M_{h} \not \subset H\right)$, it is convenient to equip $Y_{h} \subset Z:=V \times M$ with the natural norm of $Z$ and to formulate duality products using $Z$. We first treat the simpler case $\Lambda_{h}=M_{h}$.

Proposition 4.3. Assume $\alpha_{W}-k_{\psi^{\prime}} c_{M}^{2} c_{B}^{2}>0$ and $\Lambda_{h}=M_{h}$. Then, the functional $J_{r, h}$ is $\alpha$-convex on $K_{h} \cap$ ker $\tilde{B}_{h}$, namely there is $\alpha>0$ such that for all $r \geq 0$,

$$
\forall y_{h}, z_{h} \in K_{h} \cap \operatorname{ker} \tilde{B}_{h}, \quad\left\langle J_{r, h}^{\prime}\left(y_{h}\right)-J_{r, h}^{\prime}\left(z_{h}\right), y_{h}-z_{h}\right\rangle_{Z^{\prime}, Z} \geq \alpha\left\|y_{h}-z_{h}\right\|_{Z}^{2} .
$$

Proof. Let $y_{h}, z_{h} \in K_{h} \cap$ ker $\tilde{B}_{h}$ with $y_{h}:=\left(v_{h}, q_{h}\right)$ and $z_{h}:=\left(w_{h}, r_{h}\right)$. Set $A=\left\langle J_{r, h}^{\prime}\left(y_{h}\right)-J_{r, h}^{\prime}\left(z_{h}\right), y_{h}-z_{h}\right\rangle_{Z^{\prime}, Z}$. Since $\Lambda_{h}=M_{h}$, the penalty term in (4.16) vanishes for $y_{h}, z_{h} \in \operatorname{ker} \tilde{B}_{h}$. As a result,

$$
\begin{aligned}
A & =\left\langle W^{\prime}\left(v_{h}\right)-W^{\prime}\left(w_{h}\right), v_{h}-w_{h}\right\rangle_{V^{\prime}, V}+\left(\psi^{\prime}\left(q_{h}\right)-\psi^{\prime}\left(r_{h}\right), q_{h}-r_{h}\right)_{M_{h}} \\
& \geq \alpha_{W}\left\|v_{h}-w_{h}\right\|_{V}^{2}-k_{\psi^{\prime}} \sum_{F \in \mathcal{F}_{h}} \sum_{i=1}^{n_{q}} \omega_{i}^{F}\left(q_{h}\left(\xi_{i}^{F}\right)-r_{h}\left(\xi_{i}^{F}\right)\right)^{2}
\end{aligned}
$$

where we have used the $\alpha$-convexity of $W$, the Lipschitz continuity of $\psi^{\prime}$, and the fact that the weights $\omega_{i}^{F}$ are positive. Moreover, since the quadrature is at least of degree $2 k$, since $\Pi_{\Lambda_{h}} B\left(v_{h}-w_{h}\right)=q_{h}-r_{h}$ by assumption, and owing to the conformity of $V_{h}$, it is inferred that

$$
\begin{aligned}
A & \geq \alpha_{W}\left\|v_{h}-w_{h}\right\|_{V}^{2}-k_{\psi^{\prime}}\left\|q_{h}-r_{h}\right\|_{M}^{2} \\
& =\alpha_{W}\left\|v_{h}-w_{h}\right\|_{V}^{2}-k_{\psi^{\prime}}\left\|\Pi_{\Lambda_{h}} B\left(v_{h}-w_{h}\right)\right\|_{M}^{2} \\
& \geq \alpha_{W}\left\|v_{h}-w_{h}\right\|_{V}^{2}-k_{\psi^{\prime}}\left\|B\left(v_{h}-w_{h}\right)\right\|_{M}^{2} \\
& \geq\left(\alpha_{W}-k_{\psi^{\prime}} c_{M}^{2} c_{B}^{2}\right)\left\|v_{h}-w_{h}\right\|_{V}^{2},
\end{aligned}
$$

whence the conclusion readily follows since $\left\|q_{h}-r_{h}\right\|_{M} \leq c_{M} c_{B}\left\|v_{h}-w_{h}\right\|_{V}$.

Proposition 4.4. Assume $\alpha_{W}-2 k_{\psi^{\prime}} c_{M}^{2} c_{B}^{2}>0$. Then, (4.19) still holds if $r>4 k_{\psi^{\prime}}$ and if $h$ is small enough. 
Proof. Proceeding as above leads to

$$
\begin{aligned}
A & \geq \alpha_{W}\left\|v_{h}-w_{h}\right\|_{V}^{2}-k_{\psi^{\prime}}\left\|q_{h}-r_{h}\right\|_{M}^{2}+r\left\|\tilde{B}_{h}^{\sharp}\left(y_{h}-z_{h}\right)\right\|_{M}^{2} \\
& \geq \alpha_{W}\left\|v_{h}-w_{h}\right\|_{V}^{2}-2 k_{\psi^{\prime}}\left\|\Pi_{\Lambda_{h}} B\left(v_{h}-w_{h}\right)\right\|_{M}^{2}-2 k_{\psi^{\prime}}\left\|\left(I-\Pi_{\Lambda_{h}}\right)\left(q_{h}-r_{h}\right)\right\|_{M}^{2}+r\left\|\tilde{B}_{h}^{\sharp}\left(y_{h}-z_{h}\right)\right\|_{M}^{2},{ }^{2},{ }^{2},{ }^{2} \\
& =\left(\alpha_{W}-2 k_{\psi^{\prime}} c_{M}^{2} c_{B}^{2}\right)\left\|v_{h}-w_{h}\right\|_{V}^{2}-2 k_{\psi^{\prime}}\left\|\left(I-\Pi_{\Lambda_{h}}\right)\left(q_{h}-r_{h}\right)\right\|_{M}^{2}+r\left\|\Pi_{M_{h}} B\left(v_{h}-w_{h}\right)-\left(q_{h}-r_{h}\right)\right\|_{M}^{2},
\end{aligned}
$$

since $\Pi_{\Lambda_{h}} B\left(v_{h}-w_{h}\right)=\Pi_{\Lambda_{h}}\left(q_{h}-r_{h}\right)$. The last term in the right-hand side can be transformed into

$$
\begin{aligned}
\left\|\Pi_{M_{h}} B\left(v_{h}-w_{h}\right)-\left(q_{h}-r_{h}\right)\right\|_{M}^{2} & =\left\|\Pi_{M_{h}} B\left(v_{h}-w_{h}\right)-\Pi_{\Lambda_{h}} B\left(v_{h}-w_{h}\right)-\left(I-\Pi_{\Lambda_{h}}\right)\left(q_{h}-r_{h}\right)\right\|_{M}^{2} \\
& \geq \frac{1}{2}\left\|\left(I-\Pi_{\Lambda_{h}}\right)\left(q_{h}-r_{h}\right)\right\|_{M}^{2}-\left\|\Pi_{M_{h}} B\left(v_{h}-w_{h}\right)-\Pi_{\Lambda_{h}} B\left(v_{h}-w_{h}\right)\right\|_{M}^{2} \\
& \geq \frac{1}{2}\left\|\left(I-\Pi_{\Lambda_{h}}\right)\left(q_{h}-r_{h}\right)\right\|_{M}^{2}-\left\|\left(I-\Pi_{\Lambda_{h}}\right) B\left(v_{h}-w_{h}\right)\right\|_{M}^{2}
\end{aligned}
$$

since $\Lambda_{h} \subset M_{h}$. Moreover, in all cases for $\Lambda_{h}$,

$$
\left\|\left(I-\Pi_{\Lambda_{h}}\right) B\left(v_{h}-w_{h}\right)\right\|_{M} \lesssim h^{1 / 2}\left\|B\left(v_{h}-w_{h}\right)\right\|_{H} \lesssim h^{1 / 2}\left\|v_{h}-w_{h}\right\|_{V} .
$$

To conclude, observe that $\left\|\Pi_{\Lambda_{h}}\left(q_{h}-r_{h}\right)\right\|_{M}=\left\|\Pi_{\Lambda_{h}} B\left(v_{h}-w_{h}\right)\right\|_{M} \leq c_{M} c_{B}\left\|v_{h}-w_{h}\right\|_{V}$.

As in the continuous case, the discrete decomposed problem (4.18) is tackled by solving the stationarity conditions for the discrete augmented Lagrangian $L_{r, h}$, that is, we seek $x_{h}:=\left(u_{h}, p_{h}\right) \in V_{h} \times M_{h}^{+}$and $\theta_{h} \in \Lambda_{h}$ such that

$$
\begin{array}{ll}
\left\langle W^{\prime}\left(u_{h}\right), v_{h}\right\rangle_{V^{\prime}, V}+\left(\theta_{h}, B v_{h}\right)_{M}+r\left(\Pi_{M_{h}} B u_{h}-p_{h}, B v_{h}\right)_{M}=0, & \forall v_{h} \in V_{h}, \\
\left(\psi^{\prime}\left(p_{h}\right), q_{h}-p_{h}\right)_{M_{h}}-\left(\theta_{h}, q_{h}-p_{h}\right)_{M}-r\left(B u_{h}-p_{h}, q_{h}-p_{h}\right)_{M} \geq 0, & \forall q_{h} \in M_{h}^{+}, \\
\left(\lambda_{h}, B u_{h}-p_{h}\right)_{M}=0, & \forall \lambda_{h} \in \Lambda_{h} .
\end{array}
$$

By proceeding as in the continuous case (and using additional simplifications due to the finite-dimensional setting), the following equivalence result is readily verified.

Proposition 4.5. If $\left(x_{h}, \theta_{h}\right)$ is a local saddle-point of $L_{r, h}$ on $K_{h} \times \Lambda_{h}$, then $x_{h} \in K_{h} \cap$ ker $\tilde{B}_{h}$ is a local minimizer of the discrete decomposed problem (4.18). Conversely, let $x_{h} \in K_{h} \cap$ ker $\tilde{B}_{h}$ be a local minimizer of the discrete decomposed problem (4.18). Then, there exists a unique $\theta_{h} \in \Lambda_{h}$ such that $\left(x_{h}, \theta_{h}\right)$ is a stationary point of $L_{r, h}$ on $K_{h} \times \Lambda_{h}$. Moreover, if the following second-order condition holds,

$$
\left\langle J_{0, h}^{\prime \prime}\left(x_{h}\right),\left(d_{h}, d_{h}\right)\right\rangle>0, \quad \forall d_{h} \in\left(V_{h} \times \overline{C_{+, h}\left(x_{h}\right)}\right) \cap \text { ker } \tilde{B}_{h} \backslash\{0\},
$$

where $C_{+, h}\left(x_{h}\right)=\left\{d_{h} \in M_{h} ; \exists \alpha>0, p_{h}+\alpha d_{h} \in M_{h}^{+}\right\}$, then $\left(x_{h}, \theta_{h}\right)$ is a local saddle-point of the augmented Lagrangian on $K_{h} \times \Lambda_{h}$ for $r$ large enough.

Remark 4.6. In the decomposition-coordination method or when assembling the Jacobian matrix in Newton's method (see Sect. 5), the variational inequality (4.21) has to be solved with fixed $u_{h}$ and $\theta_{h}$. This amounts to a nonlinear problem of size the dimension of $M_{h}$, namely of size $n_{q} \times N_{\Gamma}$ where $n_{q}$ is defined above and where $N_{\Gamma}$ stands for the cardinal number of the set $\mathcal{F}_{h}$. The key point is that since the underlying quadrature is at least of degree $2 k,(4.21)$ is equivalent to

$$
\left(\psi^{\prime}\left(p_{h}\right), q_{h}-p_{h}\right)_{M_{h}}-\left(\theta_{h}, q_{h}-p_{h}\right)_{M_{h}}-r\left(B u_{h}-p_{h}, q_{h}-p_{h}\right)_{M_{h}} \geq 0, \quad \forall q_{h} \in M_{h}^{+},
$$


and using the nodal basis of $M_{h}$, this leads to $n_{q} \times N_{\Gamma}$ uncoupled one-dimensional nonlinear problems. Note that (4.24) amounts to the minimization problem

$$
\min _{q_{h} \in M_{h}^{+}}\left(\psi_{h}\left(q_{h}\right), 1\right)_{M_{h}}-\left(\theta_{h}, q_{h}\right)_{M}+\frac{r}{2}\left\|\tilde{B}_{h}^{\sharp}\left(y_{h}, q_{h}\right)\right\|_{M}^{2} .
$$

It is readily verified that for $r \geq k_{\psi^{\prime}}$, the above functional is convex so that the minimization problem (4.25) has a unique solution.

\subsection{Error analysis}

This section is devoted to the error analysis for the three choices (4.6)-(4.8) of discrete spaces $V_{h}, M_{h}$, and $\Lambda_{h}$. Their analysis is of increasing complexity. In (4.6) and (4.7), $\Lambda_{h}=M_{h}$, while $M_{h}^{+} \subset M^{+}$in (4.6), but $M_{h}^{+} \not \subset M^{+}$in (4.7); finally, in (4.8), $\Lambda_{h} \neq M_{h}$ and $M_{h}^{+} \not \subset M^{+}$. In all cases, the goal is to obtain error estimates with (quasi)optimal convergence rates in the mesh-size $h$ under the assumption that the exact solution is unique and smooth enough. We assume for the sake of simplicity that the functional $J_{r, h}$ is $\alpha$-convex on $K_{h} \cap \operatorname{ker} \tilde{B}_{h}$ so that the discrete solution is also unique. Sufficient conditions for $\alpha$-convexity and uniqueness are given by Propositions 4.3 and 4.4 above. In the sequel, $(x, \theta)$ with $x:=(u, p)$ denotes the exact solution and $\left(x_{h}, \theta_{h}\right)$ with $x_{h}:=\left(u_{h}, p_{h}\right)$ denotes the approximate solution. Henceforth, we assume that $\theta \in M$. Then, using the density of $H^{+}$in $M^{+},(3.9)$ yields $\left(\psi^{\prime}(p)-\theta, q-p\right)_{M} \geq 0$ for all $q \in M^{+}$, whence it is classically deduced that $\psi^{\prime}(p)-\theta \in M^{+}$and that $\operatorname{supp}\left(\psi^{\prime}(p)-\theta\right) \cap \operatorname{supp}(p)$ has zero measure.

We introduce an additional regularity assumption regarding the topology of the subset of $\Gamma$ where the unilateral constraint $p \geq 0$ is actually active, namely, letting

$$
\Gamma_{0}(p):=\{x \in \Gamma ; p(x)=0\}, \quad \text { and } \quad \Gamma_{+}(p):=\Gamma \backslash \Gamma_{0}(p),
$$

we assume that the set $\overline{\Gamma_{0}(p)} \cap \overline{\Gamma_{+}(p)}$ is

- in $2 \mathrm{D} / 1 \mathrm{D}$, a finite union of points;

- in $3 \mathrm{D} / 2 \mathrm{D}$, a finite union of Lipschitz curves.

Under this assumption, henceforth referred to as $\mathrm{A}[p]$, a sharper error estimate can be obtained by using the modified Lagrange interpolate introduced by Hüeber and Wohlmuth [19] in the piecewise affine case or its piecewise quadratic extension in 2D/1D introduced in Lemma 4.13 below.

Since we are working in a nonconforming framework $\left(M_{h} \not \subset H\right.$ and possibly $\left.M_{h}^{+} \not \subset M^{+}\right)$and recalling that we have set $Z:=V \times M$, it is convenient to redefine the operator $\tilde{B}$ as $Z \ni y:=(v, q) \mapsto B v-q \in M$ and to extend the domain of the functional $J_{r}$ to $Z$. Moreover, taking advantage that for the exact solution $\theta \in M$, the augmented Lagrangian is now redefined as

$$
L_{r}: Z \times M \ni(y, \lambda) \longmapsto L_{r}(y, \lambda):=J_{r}(y)+(\lambda, \tilde{B} y)_{M} \in \mathbb{R} .
$$

\subsubsection{An abstract error estimate}

The proof of the following key abstract error estimate is postponed to Appendix A. Observe that the error $\left(x-x_{h}\right)$ is measured in the $\|\cdot\|_{Z}$-norm, that is the $H^{1}(\Omega)^{d}$-norm for the bulk displacement and the $L^{2}(\Gamma)$-norm for the normal displacement on $\Gamma$, while the error $\left(\theta-\theta_{h}\right)$ on the Lagrange multiplier is measured in the $L^{2}(\Gamma)$-norm scaled by the factor $h^{1 / 2}$.

Lemma 4.7. For all $y_{h}:=\left(v_{h}, q_{h}\right) \in K_{h} \cap$ ker $\tilde{B}_{h}^{\sharp}$ and for all $q \in M^{+}$, letting

$$
\begin{aligned}
\eta_{\text {unil }}(q) & :=\left(\psi^{\prime}(p)-\theta, q-p_{h}\right)_{M}, \\
\eta_{\text {unil }}\left(q_{h}\right) & :=\left(\psi^{\prime}(p)-\theta, q_{h}-p\right)_{M}, \\
\eta_{\text {quad }}\left(q_{h}\right) & :=\sup _{r_{h} \in M_{h},\left\|r_{h}\right\|_{M}=1}\left|\left(\psi^{\prime}\left(q_{h}\right), r_{h}\right)_{M}-\left(\psi^{\prime}\left(q_{h}\right), r_{h}\right)_{M_{h}}\right|,
\end{aligned}
$$



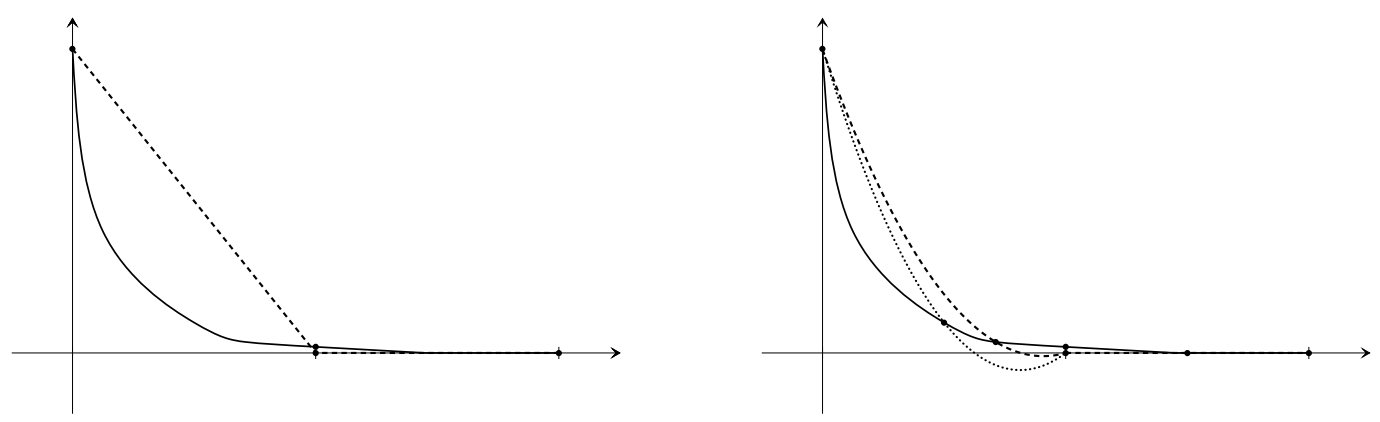

Figure 3. Principle of the Hüeber-Wohlmuth interpolate; left: piecewise affine case; right: piecewise quadratic case.

there holds

$$
\begin{aligned}
& \left\|x-x_{h}\right\|_{Z}^{2} \lesssim\left\|x-y_{h}\right\|_{Z}^{2}+\eta_{\text {unil }}\left(q_{h}\right)+\eta_{\text {quad }}\left(q_{h}\right)^{2}+\eta_{\text {unil }}(q)+h^{s}\left\|\theta-\Pi_{\Lambda_{h}} \theta\right\|_{M}^{2} \\
& \beta_{h} h^{1 / 2}\left\|\theta-\theta_{h}\right\|_{M} \lesssim h^{1 / 2}\left\|\theta-\Pi_{\Lambda_{h}} \theta\right\|_{M}+\left\|x-x_{h}\right\|_{Z}
\end{aligned}
$$

where $s=1$ if $\Lambda_{h}=M_{h}$ and $s=0$ otherwise.

Remark 4.8. $\eta_{\text {unil }}(q)$ measures the nonconformity error resulting from $M_{h}^{+} \not \subset M^{+}$; indeed, if $p_{h} \in M^{+}$, taking $q=p_{h}$ yields $\eta_{\text {unil }}(q)=0 . \quad \eta_{\text {quad }}\left(q_{h}\right)$ measures the quadrature error when evaluating the cohesive energy. Finally, $\left\|x-y_{h}\right\|_{Z}+\eta_{\text {unil }}\left(q_{h}\right)$ measures the interpolation error while accounting for the unilateral constraint.

4.3.2. The case $M_{h}=\mathcal{P}_{\mathrm{d}}^{0}\left(\mathcal{F}_{h}\right), \Lambda_{h}=M_{h}$, and $V_{h} \supset \mathcal{P}_{\mathrm{c}}^{1}\left(\mathcal{T}_{h}\right)^{d}$

Theorem 4.9. Let $M_{h}=\mathcal{P}_{\mathrm{d}}^{0}\left(\mathcal{F}_{h}\right), \Lambda_{h}=M_{h}$, and $V_{h} \supset \mathcal{P}_{\mathrm{c}}^{1}\left(\mathcal{T}_{h}\right)^{d}$. Assume $u \in H^{3 / 2+\nu}(\Omega)$ with $0<\nu \leq \frac{1}{2}$, so that $p \in H^{1+\nu}(\Gamma)$ and $\theta \in H^{\nu}(\Gamma)$. Then, in the above framework, there holds

$$
\left\|x-x_{h}\right\|_{Z}+\beta_{h} h^{1 / 2}\left\|\theta-\theta_{h}\right\|_{M} \lesssim h^{1 / 2+\nu} .
$$

Proof. We apply Lemma 4.7 in the setting $\Lambda_{h}=M_{h}$ and $\tilde{B}_{h}^{\sharp}=\tilde{B}_{h}$. Since $M_{h}^{+} \subset M^{+}$because $M_{h}=\mathcal{P}_{\mathrm{d}}^{0}\left(\mathcal{F}_{h}\right)$, we can take $q=p_{h}$ to obtain $\eta_{\text {unil }}(q)=0$. Moreover, it is readily verified that for piecewise constant functions, $\eta_{\text {quad }}\left(q_{h}\right)=0$. It remains to select $y_{h}:=\left(v_{h}, q_{h}\right) \in K_{h} \cap \operatorname{ker} \tilde{B}_{h}$ to estimate $\eta_{\text {unil }}\left(q_{h}\right)$ and $\left\|x-y_{h}\right\|_{Z}$. Let $\mathcal{I}_{\mathrm{HW}}^{1}$ be the piecewise affine interpolation operator introduced by Hüeber and Wohlmuth; see [19] and also the left panel of Figure 3. Recall that $\mathcal{I}_{\mathrm{HW}}^{1} p \geq 0$ on $\Gamma$ and that $\operatorname{supp}\left(\mathcal{I}_{\mathrm{HW}}^{1} p\right) \subset \operatorname{supp}(p)$. In particular, since $\operatorname{supp}\left(\psi^{\prime}(p)-\theta\right) \cap \operatorname{supp}(p)$ has zero measure, it is inferred that $\left(\psi^{\prime}(p)-\theta, \mathcal{I}_{\mathrm{HW}}^{1} p\right)_{M}=0$. Hence, setting $q_{h}:=\Pi_{M_{h}} \mathcal{I}_{\mathrm{HW}}^{1} p$, it is clear that $q_{h} \in M_{h}^{+}$since $M_{h}=\mathcal{P}_{\mathrm{d}}^{0}\left(\mathcal{F}_{h}\right)$. Moreover, observing that $q_{h}$ and $\mathcal{I}_{\mathrm{HW}}^{1} p$ have the same support yields

$$
\eta_{\text {unil }}\left(q_{h}\right)=0
$$

Now, let $\mathcal{I}_{\text {Lag }}^{1}$ be the usual piecewise affine Lagrange interpolation operator (the same notation is used for interpolating vector-valued functions in $\Omega$ and scalar-valued functions on $\Gamma$ ). Define $v_{h} \in \mathcal{P}_{\mathrm{c}}^{1}\left(\mathcal{T}_{h}\right)^{d}$ from $\mathcal{I}_{\text {Lag }}^{1} u$ by just modifying the normal component of the nodal values located on $\Gamma$ so that $B v_{h}=\mathcal{I}_{\mathrm{HW}}^{1} p$ on $\Gamma$. Then, by construction, $y_{h}:=\left(v_{h}, q_{h}\right) \in K_{h} \cap$ ker $\tilde{B}_{h}$. In addition, since $u \in H^{3 / 2+\nu}(\Omega)$, standard interpolation properties (see, e.g., [12]) lead to

$$
\left\|u-\mathcal{I}_{\text {Lag }}^{1} u\right\|_{V} \lesssim h^{1 / 2+\nu}
$$


and using an inverse inequality, the triangle inequality, standard approximation properties of $\mathcal{I}_{\text {Lag, }}^{1}$, and the fact that $p \in H^{1+\nu}(\Gamma)$ yields

$$
\left\|\mathcal{I}_{\text {Lag }}^{1} u-v_{h}\right\|_{V} \lesssim h^{-1 / 2}\left\|\mathcal{I}_{\text {Lag }}^{1} p-\mathcal{I}_{\mathrm{HW}}^{1} p\right\|_{M} \leq h^{-1 / 2}\left(h^{1+\nu}+\left\|p-\mathcal{I}_{\mathrm{HW}}^{1} p\right\|_{M}\right) .
$$

Assumption $\mathrm{A}[p]$ is now used to infer that $\left\|p-\mathcal{I}_{\mathrm{HW}}^{1} p\right\|_{M} \lesssim h^{1+\nu}$; see [19]. Collecting the above estimates yields $\left\|u-v_{h}\right\|_{V} \lesssim h^{1 / 2+\nu}$ and since

$$
\left\|p-q_{h}\right\|_{M} \leq\left\|p-\Pi_{M_{h}} p\right\|_{M}+\left\|\Pi_{M_{h}}\left(p-\mathcal{I}_{\mathrm{HW}}^{1} p\right)\right\|_{M} \leq\left\|p-\Pi_{M_{h}} p\right\|_{M}+\left\|p-\mathcal{I}_{\mathrm{HW}}^{1} p\right\|_{M} \lesssim h,
$$

and recalling that $\nu \leq \frac{1}{2}$, it is inferred that

$$
\left\|x-y_{h}\right\|_{Z} \lesssim h^{1 / 2+\nu} .
$$

Finally, since $\theta \in H^{\nu}(\Gamma),\left\|\theta-\Pi_{\Lambda_{h}} \theta\right\|_{M} \lesssim h^{\nu}$, whence the conclusion is straightforward.

Remark 4.10. Without assumption $\mathrm{A}[p], \eta_{\text {unil }}\left(q_{h}\right)$ can be estimated by taking $q_{h}=\Pi_{M_{h}} \mathcal{I}_{\text {Lag }}^{1} p$. Since $\psi^{\prime}$ is Lipschitz continuous and $\nu \leq \frac{1}{2}, \zeta:=\psi^{\prime}(p)-\theta \in H^{\nu}(\Gamma)$. Hence, $\eta_{\text {unil }}\left(q_{h}\right) \leq\left\|\zeta-\Pi_{M_{h}} \zeta\right\|_{M}\left\|p-\Pi_{M_{h}} \mathcal{I}_{\text {Lag }}^{1} p\right\|_{M} \lesssim$ $h^{\nu}\left\|p-\Pi_{M_{h}} \mathcal{I}_{\text {Lag }}^{1} p\right\|_{M} \lesssim h^{1+\nu}$, so that the upper bound in (4.33) becomes $h^{1 / 2+\nu / 2}$.

4.3.3. The case $M_{h}=\mathcal{P}_{\mathrm{d}}^{1}\left(\mathcal{F}_{h}\right), \Lambda_{h}=M_{h}$, and $V_{h} \supset \mathcal{P}_{\mathrm{c}}^{2}\left(\mathcal{T}_{h}\right)^{d}$

Theorem 4.11. Let $M_{h}=\mathcal{P}_{\mathrm{d}}^{1}\left(\mathcal{F}_{h}\right), \Lambda_{h}=M_{h}$, and $V_{h} \supset \mathcal{P}_{\mathrm{c}}^{2}\left(\mathcal{T}_{h}\right)^{d}$. Assume $u \in H^{2+\nu}(\Omega)$ with $0<\nu \leq \frac{1}{2}$, so that $p \in H^{3 / 2+\nu}(\Gamma)$ and $\theta \in H^{1 / 2+\nu}(\Gamma)$. Then, in the above framework, there holds in $3 D / 2 D$,

$$
\left\|x-x_{h}\right\|_{Z}+\beta_{h} h^{1 / 2}\left\|\theta-\theta_{h}\right\|_{M} \lesssim h^{3 / 4+\nu / 2} .
$$

Moreover, in $2 D / 1 D$, if $\psi$ is of class $\mathrm{C}^{2}$ with $\psi^{\prime \prime}$ Lipschitz continuous,

$$
\left\|x-x_{h}\right\|_{Z}+\beta_{h} h^{1 / 2}\left\|\theta-\theta_{h}\right\|_{M} \lesssim h^{1+\nu} .
$$

Proof. Again, we apply Lemma 4.7 in the setting $\Lambda_{h}=M_{h}$ and $\tilde{B}_{h}^{\sharp}=\tilde{B}_{h}$. Consider first $\eta_{\text {unil }}(q)$. Take $q=\Pi_{0} p_{h}$, where $\Pi_{0}$ denotes the $M$-orthogonal projection onto $\mathcal{P}_{\mathrm{d}}^{0}\left(\mathcal{F}_{h}\right)$, and observe that $q \in M^{+}$. Letting $\zeta:=\psi^{\prime}(p)-\theta$, this yields

$$
\begin{aligned}
\eta_{\text {unil }}(q) & =\left(\zeta, \Pi_{0} p_{h}-p_{h}\right)_{M}=\left(\zeta-\Pi_{0} \zeta, \Pi_{0} p_{h}-p_{h}\right)_{M} \\
& =\left(\zeta-\Pi_{0} \zeta, \Pi_{0} p_{h}-\Pi_{0} p+\Pi_{0} p-p+p-p_{h}\right)_{M} \\
& \lesssim\left\|\zeta-\Pi_{0} \zeta\right\|_{M}\left(h^{1 / 2}\left\|p-p_{h}\right\|_{H}+\left\|\Pi_{0} p-p\right\|_{M}\right),
\end{aligned}
$$

where the $H$-stability of $\Pi_{0}$ has been used owing to the quasi-uniformity of the mesh on $\Gamma$ and since $\left\|\zeta-\Pi_{0} \zeta\right\|_{H^{\prime}} \lesssim$ $h^{1 / 2}\left\|\zeta-\Pi_{0} \zeta\right\|_{M}$. Moreover,

$$
\begin{aligned}
\left\|p-p_{h}\right\|_{H} & =\left\|B u-\Pi_{\Lambda_{h}} B u_{h}\right\|_{H} \leq\left\|B u-\Pi_{\Lambda_{h}} B u\right\|_{H}+\left\|\Pi_{\Lambda_{h}}\left(B u-B u_{h}\right)\right\|_{H} \\
& \lesssim\left\|B u-\Pi_{\Lambda_{h}} B u\right\|_{H}+\left\|B u-B u_{h}\right\|_{H} \lesssim h^{1+\nu}+\left\|u-u_{h}\right\|_{V} .
\end{aligned}
$$

Since $\psi^{\prime}$ is Lipschitz continuous and $\nu \leq \frac{1}{2}$, classical properties of superposition operators [26] yield $\zeta \in$ $H^{1 / 2+\nu}(\Gamma)$, so that $\left\|\zeta-\Pi_{0} \zeta\right\|_{M} \lesssim h^{1 / 2+\nu}$. Moreover, $\left\|\Pi_{0} p-p\right\|_{M} \lesssim h$ since $p \in H^{1}(\Gamma)$. Hence,

$$
\eta_{\text {unil }}(q) \lesssim h^{1+\nu}\left\|u-u_{h}\right\|_{V}+h^{3 / 2+\nu} .
$$


Consider now $\eta_{\text {quad }}\left(q_{h}\right)$ for $q_{h} \in M_{h}$. Since the quadrature is at least of degree 2 , letting $\mathcal{I}_{\xi}^{1}$ be the (discontinuous) interpolation operator at the Gauss nodes, there holds

$$
\eta_{\text {quad }}\left(q_{h}\right) \leq\left\|\psi^{\prime}\left(q_{h}\right)-\mathcal{I}_{\xi}^{1}\left(\psi^{\prime}\left(q_{h}\right)\right)\right\|_{M} .
$$

Since $\psi^{\prime}$ is Lipschitz continuous, $\psi^{\prime}\left(q_{h}\right)$ is in $H^{1}\left(\mathcal{F}_{h}\right)$, the usual broken Sobolev space on the mesh $\mathcal{F}_{h}$, and there holds $\left\|\psi^{\prime}\left(q_{h}\right)\right\|_{H^{1}\left(\mathcal{F}_{h}\right)} \lesssim\left\|q_{h}\right\|_{H^{1}\left(\mathcal{F}_{h}\right)}$. Hence,

$$
\eta_{\text {quad }}\left(q_{h}\right) \lesssim h\left\|q_{h}\right\|_{H^{1}\left(\mathcal{F}_{h}\right)} .
$$

Consider now $\eta_{\text {unil }}\left(q_{h}\right)$ and $\left\|x-y_{h}\right\|_{Z}$. In $3 \mathrm{D} / 2 \mathrm{D}$, we set $v_{h}=\mathcal{I}_{\text {Lag }}^{2} u$, the piecewise quadratic Lagrange interpolate of $u$, and $q_{h}=\Pi_{M_{h}} \mathcal{I}_{\text {Lag }}^{2} p$. Then, $q_{h} \in M_{h}^{+}$; see Lemma 4.12 below. Moreover,

$$
\eta_{\text {unil }}\left(q_{h}\right) \lesssim\left\|p-q_{h}\right\|_{M} \lesssim h^{3 / 2+\nu},
$$

and $\left\|x-y_{h}\right\|_{Z} \lesssim h^{1+\nu}$. Finally, $\left\|\theta-\Pi_{\Lambda_{h}} \theta\right\|_{M} \lesssim h^{1 / 2+\nu}$. Collecting the above estimates yields (4.34).

In $2 \mathrm{D} / 1 \mathrm{D}$, we consider the piecewise quadratic extension, $\mathcal{I}_{\mathrm{HW}}^{2}$, of the Hüeber-Wohlmuth interpolation operator; see Lemma 4.13 below. Then, we set $q_{h}=\Pi_{M_{h}} \mathcal{I}_{\mathrm{HW}}^{2} p$ and $v_{h}$ is obtained from $\mathcal{I}_{\text {Lag }}^{2} u$ by just modifying the normal component of the nodal values located on $\Gamma$ so that $B v_{h}=\mathcal{I}_{\mathrm{HW}}^{2} p$. Then, proceeding as in the proof of Theorem 4.9 yields $\eta_{\text {unil }}\left(q_{h}\right)=0$, and $\left\|x-y_{h}\right\|_{Z} \lesssim h^{1+\nu}$. Furthermore, owing to the assumption on $\psi$ and observing that $\left(\psi^{\prime}\left(q_{h}\right)\right)^{\prime}=\psi^{\prime \prime}\left(q_{h}\right) q_{h}^{\prime}$, it is readily seen using inverse inequalities and the fact that $q_{h}$ is piecewise affine that $\left\|\psi^{\prime}\left(q_{h}\right)\right\|_{H^{3 / 2}\left(\mathcal{F}_{h}\right)} \lesssim h^{-1 / 2}\left\|q_{h}\right\|_{H^{1}\left(\mathcal{F}_{h}\right)}^{2}$. Hence,

$$
\eta_{\text {quad }}\left(q_{h}\right) \lesssim h^{3 / 2}\left\|q_{h}\right\|_{H^{1}\left(\mathcal{F}_{h}\right)}^{2} .
$$

Finally, still using assumption $\mathrm{A}[p]$, the above estimate on $\eta_{\text {unil }}(q)$ can be sharpened using the fact that (see [18], Thm. 4.4) $\left(\zeta-\Pi_{0} \zeta, \Pi_{0} p-p\right)_{M} \lesssim h^{2+2 \nu}$ yielding

$$
\eta_{\mathrm{unil}}(q) \lesssim h^{1+\nu}\left\|u-u_{h}\right\|_{V}+h^{2+2 \nu} .
$$

Collecting the above estimates yields (4.35).

Lemma 4.12. Let $F$ be a triangle, let $u \in \mathbb{P}_{2}(F)$, and assume that $u$ is non-negative at the six interpolation nodes of $\mathcal{I}_{\text {Lag }}^{2}$ in $F$. Let $\Pi_{1} u$ be the $L^{2}$-orthogonal projection of $u$ onto $\mathbb{P}_{1}(F)$. Let $\left(\xi_{i}^{F}\right)_{1 \leq i \leq 3}$ be the midpoints of the three edges of $F$. Then, for all $1 \leq i \leq 3, \Pi_{1} u\left(\xi_{i}^{F}\right) \geq 0$.

Proof. Let $\left(\phi_{i}^{F}\right)_{1 \leq i \leq 3}$ be the (Crouzeix-Raviart) basis functions associated with the nodes $\left(\xi_{i}^{F}\right)_{1 \leq i \leq 3}$. Observe that for all $1 \leq i \leq 3$,

$$
\frac{1}{3} \Pi_{1} u\left(\xi_{i}^{F}\right)=\frac{1}{|F|} \int_{F} u \phi_{i}^{F} .
$$

Moreover, using the classical 7-point quadrature of degree 3 yields

$$
\int_{F} u \phi_{1}^{F}=\frac{3}{20} u\left(\gamma^{F}\right)+\frac{2}{15} u\left(\xi_{1}^{F}\right)+\frac{1}{20}\left(u\left(\sigma_{2}^{F}\right)+u\left(\sigma_{3}^{F}\right)-u\left(\sigma_{1}^{F}\right)\right),
$$

where $\gamma^{F}$ denotes the barycenter of $F$ and $\sigma_{i}^{F}$ is the vertex of $F$ opposite the edge containing $\xi_{i}^{F}$. Furthermore, since $u \in \mathbb{P}_{2}(F)$,

$$
u\left(\gamma^{F}\right)=\frac{1}{3}\left(u\left(\sigma_{1}^{F}\right)+u\left(\sigma_{2}^{F}\right)+u\left(\sigma_{3}^{F}\right)\right)+\frac{4}{9}\left(u\left(\xi_{1}^{F}\right)+u\left(\xi_{2}^{F}\right)+u\left(\xi_{3}^{F}\right)\right),
$$

so that $\int_{F} u \phi_{1}^{F}$ is a linear combination of non-negative terms. The same holds for $i \in\{2,3\}$. 
Lemma 4.13. Assume $\Omega \subset \mathbb{R}^{2}$. Then, there exists an interpolation operator $\mathcal{I}_{\mathrm{HW}}^{2}: H^{1}(\Gamma) \rightarrow \mathcal{P}_{\mathrm{c}}^{2}\left(\mathcal{F}_{h}\right)$ such that

(i) if $q \geq 0$, then $\mathcal{I}_{\mathrm{HW}}^{2} q\left(\xi_{i}^{F}\right) \geq 0$ for all $1 \leq i \leq n_{q}$ and for all $F \in \mathcal{F}_{h}$;

(ii) $\operatorname{supp}\left(\mathcal{I}_{\mathrm{HW}}^{2} q\right) \subset \operatorname{supp}(q)$;

(iii) if $p \in H^{3 / 2+\nu}(\Gamma), \nu \leq \frac{3}{2}$, satisfies assumption $\mathrm{A}[p]$, then $\left\|p-\mathcal{I}_{\mathrm{HW}}^{2} p\right\|_{M} \lesssim h^{3 / 2+\nu}$.

Proof. Consider first the usual, piecewise quadratic Lagrange interpolate of $q$, say $\mathcal{I}_{\text {Lag }}^{2} q$. Since $\Omega \subset \mathbb{R}^{2}$, each $F \in \mathcal{F}_{h}$ is a segment and the restriction of $\mathcal{I}_{\text {Lag }}^{2} q$ to $F$ can be specified by its values at the endpoints and the midpoint of $F$. Two modifications need to be implemented. Firstly, as for $\mathcal{I}_{\text {HW }}^{1}$, if for $F \in \mathcal{F}_{h}$, $F \cap\{x \in \Gamma ; q(x)=0\}$ has nonzero measure, the three nodal values of $\mathcal{I}_{\mathrm{HW}}^{2} q$ are set to zero on $F$. This suffices to guarantee property (ii). However, contrary to the construction of $\mathcal{I}_{\mathrm{HW}}^{1}$, this does not suffice to guarantee property (i). To this purpose a further modification is needed. Let $F \in \mathcal{F}_{h}$ and assume that $\mathcal{I}_{\text {Lag }}^{2} q$ is negative at one quadrature node (it cannot take negative values at the two quadrature nodes since it takes non-negative values at the interval endpoints and midpoint and can have only one minimizer). Then, instead of interpolating at the midpoint, we interpolate at this quadrature node; see the right panel of Figure 3 . To verify that the resulting quadratic interpolate is still non-negative at the other quadrature node, consider the reference interval $[0,1]$ with $\xi_{ \pm}=\frac{1}{2}\left(1 \pm \frac{1}{\sqrt{3}}\right)$. Let $\lambda_{0}(t)=1-t, \lambda_{1}(t)=t$, and $b(t)=4 t(1-t)$ so that the usual quadratic Lagrange interpolate of a non-negative function $q$ takes the form

$$
\alpha \lambda_{0}(t)+\beta \lambda_{1}(t)+\left(\gamma-\frac{1}{2}(\alpha+\beta)\right) b(t)
$$

with non-negative coefficients $\alpha=q(0), \beta=q(1)$, and $\gamma=q\left(\frac{1}{2}\right)$. If this interpolate is negative at, say, $\xi_{+}$, this yields $\frac{1}{6}(\alpha+\beta)+\frac{2}{3} \gamma-\frac{1}{2 \sqrt{3}}(\alpha-\beta) \leq 0$, so that $\alpha \geq \beta$. Now, the Lagrange interpolate using the two endpoints and the node $\xi_{+}$takes the form

$$
\alpha \lambda_{0}(t)+\beta \lambda_{1}(t)+\frac{3}{2}\left(\delta-\alpha \lambda_{0}\left(\xi_{+}\right)-\beta \lambda_{1}\left(\xi_{+}\right)\right) b(t)
$$

with $\delta=q\left(\xi_{+}\right)$and evaluating this expression at $\xi_{-}$yields $\delta+\frac{1}{\sqrt{3}}(\alpha-\beta) \geq 0$. This proves property (i). Finally, property (iii) is established as in [19] using assumption $\mathrm{A}[p]$ and the fact that a possible interpolation at a quadrature node and not at a midpoint does not alter the asymptotic accuracy of the quadratic Lagrange interpolate.

Remark 4.14. Similar error estimates have been obtained for quadratic approximations of two-field formulations of unilateral contact problems in [18]. Estimate (4.34) is suboptimal and does not use assumption A[p]. Instead, estimate (4.35) is optimal and relies, in particular, on this latter assumption. Without this assumption, estimate (4.34) holds in $2 \mathrm{D} / 1 \mathrm{D}$; the only modification of the above proof in $3 \mathrm{D} / 2 \mathrm{D}$ is in the choice of $q_{h} \in M_{h}^{+}$to estimate $\eta_{\text {unil }}\left(q_{h}\right)$, where the Lagrange interpolation can be performed at a quadrature node instead of at the edge midpoint, similarly to the proof of Lemma 4.13. This remark also applies to the conclusions of Theorem 4.15 below.

4.3.4. The case $M_{h}=\mathcal{P}_{\mathrm{d}}^{1}\left(\mathcal{F}_{h}\right), \Lambda_{h}=\mathcal{P}_{\mathrm{c}}^{1}\left(\mathcal{F}_{h}\right)$, and $V_{h}=\mathcal{P}_{\mathrm{c}}^{2}\left(\mathcal{T}_{h}\right)^{d}$

Theorem 4.15. Let $M_{h}=\mathcal{P}_{\mathrm{d}}^{1}\left(\mathcal{F}_{h}\right), \Lambda_{h}=\mathcal{P}_{\mathrm{c}}^{1}\left(\mathcal{F}_{h}\right)$, and $V_{h}=\mathcal{P}_{\mathrm{c}}^{2}\left(\mathcal{T}_{h}\right)^{d}$. Assume $u \in H^{2+\nu}(\Omega)$ with $0<\nu \leq \frac{1}{2}$, so that $p \in H^{3 / 2+\nu}(\Gamma)$, and also assume $\theta \in H^{1+\nu}(\Gamma)$. Then, in the above framework, there holds in $3 D / 2 D$,

$$
\left\|x-x_{h}\right\|_{Z}+\beta_{h} h^{1 / 2}\left\|\theta-\theta_{h}\right\|_{M} \lesssim h^{3 / 4+\nu / 2} .
$$

Moreover, in 2D/1D, if $\psi$ is of class $\mathrm{C}^{2}$ with $\psi^{\prime \prime}$ Lipschitz continuous,

$$
\left\|x-x_{h}\right\|_{Z}+\beta_{h} h^{1 / 2}\left\|\theta-\theta_{h}\right\|_{M} \lesssim h^{1+\nu} .
$$

Proof. We apply Lemma 4.7 in the setting $\Lambda_{h} \neq M_{h}$. We proceed as above, except for the interpolation error on the Lagrange multiplier for which there holds $\left\|\theta-\Pi_{\Lambda_{h}} \theta\right\|_{M} \lesssim h^{1+\nu}$ since $\theta \in H^{1+\nu}(\Gamma)$. 


\section{Algorithms}

In this section, we present two iterative algorithms for solving the discrete problem (4.20)-(4.22), namely a decomposed version of a classical Uzawa algorithm and a nonsmooth Newton's method.

\subsection{A decomposed Uzawa algorithm}

The first algorithm we propose is a decomposed version of a classical iterative Uzawa algorithm. Each iteration of the decomposed algorithm consists of the following steps:

$$
\begin{aligned}
& u_{h}^{n+1} \leftarrow \arg \min _{v_{h} \in V_{h}} L_{r, h}\left(v_{h}, p_{h}^{n} ; \theta_{h}^{n}\right), \\
& p_{h}^{n+1} \leftarrow \arg \min _{q_{h} \in M_{h}^{+}} L_{r, h}\left(u_{h}^{n+1}, q_{h} ; \theta_{h}^{n}\right), \\
& \theta_{h}^{n+1} \leftarrow \theta_{h}^{n}+\rho \Pi_{\Lambda_{h}}\left(\Pi_{M_{h}} B_{h} u_{h}^{n+1}-p_{h}^{n+1}\right),
\end{aligned}
$$

where $\rho$ is an arbitrary positive parameter. Instead of minimizing directly the Lagrangian with respect to the couple $\left(u_{h}^{n}, p_{h}^{n}\right)$, the Lagrangian is minimized with respect to $u_{h}^{n}$ and then with respect to $p_{h}^{n}$. This is the difference with the classical Uzawa algorithm.

Step (5.1) amounts to the solution of a linear system. Moreover, at each iteration, only the right-hand side changes. Hence, this step can be efficiently solved once a factorization method has been initially performed (LU factorization for instance). Step (5.2) is solved by the collocation method described in Section 4.1. Finally, Step (5.3) is straightforward if $\Lambda_{h}=M_{h}$ since it can be performed elementwise. Otherwise, it requires a projection onto $\Lambda_{h}$ by inverting a global mass matrix.

Remark 5.1. This algorithm corresponds to ALG2 in [13], where a slightly different version called ALG3, equivalent to the LATIN method [23], is also proposed.

We now prove a global convergence result for the decomposed Uzawa algorithm in the case where $\Lambda_{h}=M_{h}$ and under the convexity condition (2.4). Recall that this condition implies the $\alpha$-convexity of the discrete functional $J_{r, h}$ and thus the uniqueness of the discrete solution $\left(u_{h}, p_{h}, \theta_{h}\right)$. The following proposition yields sufficient conditions on the parameters $r$ and $\rho$ for global convergence. Its proof is postponed to Appendix B.

Proposition 5.2. Assume $\Lambda_{h}=M_{h}$ and the convexity condition $\alpha_{W}-2 k_{\psi^{\prime}} c_{M}^{2} c_{B}^{2}>0$. If the parameters $r$ and $\rho$ are such that

$$
r \geq \rho \geq 4 k_{\psi^{\prime}},
$$

algorithm (5.1)-(5.3) is well-defined and converges to the discrete solution $\left(u_{h}, p_{h}, \theta_{h}\right)$ for any initialization.

Remark 5.3. When the functional is nonconvex, a convergence result exists for the classical Uzawa algorithm [3]. We did not try to extend it to the decomposed version described here.

\subsection{A nonsmooth Newton's method}

Let $\left(u_{h}, p_{h}, \theta_{h}\right)$ solve (4.20)-(4.22). Given $u_{h}$ and $\theta_{h}$, the variational inequality (4.21) is equivalent to the minimization problem (4.25). Recall that for $r \geq k_{\psi^{\prime}}$, this problem has a unique solution. Hence, $p_{h}$ can be expressed as a function of $u_{h}$ and $\theta_{h}$ in the form $p_{h}:=\pi\left(u_{h}, \theta_{h}\right)$. Equations (4.20) and (4.22) can then be recast as a nonlinear system of equations

$$
\begin{array}{ll}
\left\langle W^{\prime}\left(u_{h}\right), v_{h}\right\rangle_{V^{\prime}, V}+\left(\theta_{h}, v_{h}\right)_{M}+r\left(B u_{h}-\pi\left(u_{h}, \theta_{h}\right), B v_{h}\right)_{M}=0, & \forall v_{h} \in V_{h}, \\
\left(\lambda_{h}, B u_{h}-\pi\left(u_{h}, \theta_{h}\right)\right)_{M}=0, & \forall \lambda_{h} \in \Lambda_{h},
\end{array}
$$

or in a compact form with $z_{h}:=\left(u_{h}, \theta_{h}\right)$,

$$
F\left(z_{h}\right)=0,
$$


where $F$ maps $V_{h} \times \Lambda_{h}$ onto $V_{h} \times \Lambda_{h}$. Equation (5.7) can be solved using a Newton's method. However, the function $F$ is continuous but non-differentiable owing to the unilateral constraint. $F$ is locally Lipschitz continuous though, so that, according to Rademacher's theorem, it is differentiable almost everywhere. Denote by $D_{F} \subset V_{h} \times \Lambda_{h}$ the set where $F$ is differentiable. For the points where $F$ is non-differentiable, we introduce the generalized Jacobian as defined in [9],

$$
\partial F(z)=c o\left\{\lim _{z_{i} \rightarrow z, z_{i} \in D_{F}} \nabla F\left(z_{i}\right)\right\}
$$

where co stands for the convex hull of a set. The nonsmooth Newton's method consists of the step

$$
z_{h}^{n+1} \leftarrow z_{h}^{n}-G_{n}^{-1} F\left(z_{h}^{n}\right),
$$

where $G_{n} \in \partial F\left(z_{h}^{n}\right)$.

Proposition 5.4. Let $\left(u_{h}, \theta_{h}\right)$ solve (5.7). Assume that all $G \in \partial F\left(u_{h}, \theta_{h}\right)$ are nonsingular. Then, if $r \geq k_{\psi^{\prime}}$, Newton's method (5.9) is well-defined and converges to $\left(u_{h}, \theta_{h}\right)$ for any initialization sufficiently close to $\left(u_{h}, \theta_{h}\right)$.

Proof. The function $F$ is said to be semi-smooth at a point $z \in V_{h} \times \Lambda_{h}$ if $F$ is locally Lipschitz at $z$ and

$$
\lim _{G \in \partial F(z+t d), d \rightarrow e, t \rightarrow 0^{+}} G d \text { exists for all } e \in V_{h} \times \Lambda_{h} .
$$

We can verify that $F$ is semi-smooth on $V_{h} \times \Lambda_{h}$ and conclude using a convergence result [28] on Newton's method for semi-smooth functions.

Remark 5.5. Newton's method is a local method. To globalize it, a line-search technique with the augmented Lagrangian as merit function can be used.

\section{Numerical RESUlts}

To investigate numerically the proposed methodology, we build a 2D benchmark problem with analytical solution. Let $\Omega:=] 0, L_{x}[\times] 0, L_{y}\left[, \partial \Omega_{D}:=\left\{L_{x}\right\} \times\right] 0, L_{y}[$ and $\Gamma:=] 0, L_{x}[\times\{0\}$. Consider the cohesive law

$$
t(\delta)= \begin{cases}\sigma_{c} \frac{\left(\delta-d_{c}\right)^{2}}{d_{c}^{2}} & \text { if } \delta \in\left[0, d_{c}\right] \\ 0 & \text { if } \delta>d_{c} .\end{cases}
$$

Let $n \geq 2$ and let $L_{0} \in \mathbb{R}$ be such that $0<L_{0}<L_{x}$. Introducing the functions

$$
s(x)=\left\{\begin{array}{ll}
d_{c}\left(1-\frac{x}{L_{0}}\right)^{n} & \text { if } x \in\left[0, L_{0}\right], \\
0 & \text { if } x \in\left[L_{0}, L_{x}\right],
\end{array} \quad \text { and } \quad \phi(x)= \begin{cases}t(s(x)) & \text { if } x \in\left[0, L_{0}\right], \\
\sigma_{c} & \text { if } x \in\left[L_{0}, L_{x}\right],\end{cases}\right.
$$

the bulk displacement in the analytical solution is set to

$$
u(x, y):=\left(\begin{array}{c}
-y s^{\prime}(x) \\
s(x)+y \phi(x)
\end{array}\right) .
$$

This solution is built to satisfy the boundary condition of unilateral contact with cohesive forces on $\Gamma$. The normal displacement on $\Gamma$ is given by the function $s$, and the contact zone corresponds to the interval $\left[L_{0}, L_{x}\right]$. To complete the specification of the test case, it remains to choose suitable external load and boundary conditions on $\partial \Omega \backslash\left(\Gamma \cup \partial \Omega_{D}\right)$ according to (6.3). We can readily verify that $u \in H^{n-1}(\Omega)$. We also observe that $u_{\Gamma}$ does not exceed $d_{c}$ so that the regularity of $t$ at $d_{c}$ does not limit the regularity of $u$. 
TABLE 1. Errors and convergence rates with $V_{h}=\mathcal{P}_{\mathrm{c}}^{1}\left(\mathcal{T}_{h}\right)^{d}$ and $M_{h}=\Lambda_{h}=\mathcal{P}_{\mathrm{d}}^{0}\left(\mathcal{F}_{h}\right)$.

\begin{tabular}{|c|cc|cc|cc|}
\hline$h$ & \multicolumn{2}{|c|}{$\left\|u-u_{h}\right\|_{H^{1}}$} & \multicolumn{2}{|c|}{$\left\|p-p_{h}\right\|_{L^{2}}$} & \multicolumn{2}{c|}{$\left\|\theta-\theta_{h}\right\|_{L^{2}}$} \\
\hline 2.83 & $6.42 \mathrm{e}-2$ & - & $2.95 \mathrm{e}-2$ & - & $1.04 \mathrm{e}-2$ & - \\
1.54 & $2.49 \mathrm{e}-2$ & 1.55 & $1.51 \mathrm{e}-2$ & 1.09 & $5.93 \mathrm{e}-3$ & 0.92 \\
0.80 & $1.02 \mathrm{e}-2$ & 1.36 & $7.60 \mathrm{e}-3$ & 1.04 & $1.17 \mathrm{e}-3$ & 2.46 \\
0.40 & $4.98 \mathrm{e}-3$ & 1.04 & $3.80 \mathrm{e}-3$ & 1.01 & $2.50 \mathrm{e}-4$ & 2.25 \\
0.21 & $2.33 \mathrm{e}-3$ & 1.13 & $1.90 \mathrm{e}-3$ & 1.04 & $5.35 \mathrm{e}-5$ & 2.31 \\
\hline
\end{tabular}

TABLE 2. Errors and convergence rates with $V_{h}=\mathcal{P}_{\mathrm{c}}^{2}\left(\mathcal{T}_{h}\right)^{d}$ and $M_{h}=\Lambda_{h}=\mathcal{P}_{\mathrm{d}}^{1}\left(\mathcal{F}_{h}\right)$.

\begin{tabular}{|c|cc|cc|cc|}
\hline$h$ & \multicolumn{2}{|c|}{$\left\|u-u_{h}\right\|_{H^{1}}$} & \multicolumn{2}{|c|}{$\left\|p-p_{h}\right\|_{L^{2}}$} & \multicolumn{2}{c|}{$\left\|\theta-\theta_{h}\right\|_{L^{2}}$} \\
\hline 2.83 & $4.12 \mathrm{e}-3$ & - & $3.53 \mathrm{e}-3$ & - & $2.94 \mathrm{e}-4$ & - \\
1.54 & $8.60 \mathrm{e}-4$ & 2.57 & $8.87 \mathrm{e}-4$ & 2.26 & $1.10 \mathrm{e}-5$ & 5.38 \\
0.80 & $1.90 \mathrm{e}-4$ & 2.29 & $2.22 \mathrm{e}-4$ & 2.10 & $2.95 \mathrm{e}-6$ & 2.00 \\
0.40 & $5.31 \mathrm{e}-5$ & 1.86 & $5.59 \mathrm{e}-5$ & 2.01 & $7.52 \mathrm{e}-7$ & 1.99 \\
0.21 & $1.60 \mathrm{e}-5$ & 1.80 & $1.48 \mathrm{e}-5$ & 1.99 & $1.93 \mathrm{e}-7$ & 2.03 \\
\hline
\end{tabular}

TABLE 3. Number of iterations in the decomposed Uzawa algorithm for various couples $(r, \rho)$.

\begin{tabular}{|c|c|c|c|c|c|}
\hline$\rho / r$ & $r=k_{\psi^{\prime}}$ & $r=2 k_{\psi^{\prime}}$ & $r=10 k_{\psi^{\prime}}$ & $r=20 k_{\psi^{\prime}}$ & $r=100 k_{\psi^{\prime}}$ \\
\hline 0.5 & $\infty$ & 13 & 12 & 9 & 5 \\
1.0 & $\infty$ & 32 & 7 & 6 & 3 \\
1.5 & $\infty$ & $\infty$ & 11 & 7 & 5 \\
2.0 & $\infty$ & $\infty$ & $\infty$ & $\infty$ & $\infty$ \\
\hline
\end{tabular}

First, we study the convergence rates for two choices of finite element spaces, namely $V_{h}=\mathcal{P}_{\mathrm{c}}^{1}\left(\mathcal{T}_{h}\right)^{d}$ and $M_{h}=\Lambda_{h}=\mathcal{P}_{\mathrm{d}}^{0}\left(\mathcal{F}_{h}\right)$ (referred to as $\mathbb{P}_{1} / \mathbb{P}_{0}$ case) and $V_{h}=\mathcal{P}_{\mathrm{c}}^{2}\left(\mathcal{T}_{h}\right)^{d}$ and $M_{h}=\Lambda_{h}=\mathcal{P}_{\mathrm{d}}^{1}\left(\mathcal{F}_{h}\right)$ (referred to as $\mathbb{P}_{2} / \mathbb{P}_{1}$ case). The simulations are performed with the decomposed Uzawa algorithm. The Young modulus and the Poisson ratio are $E=2 \times 10^{5}$ and $\nu=0.2$. Further parameters are $L_{x}=20, L_{y}=6, L_{0}=10, \sigma_{c}=0.001$, and $d_{c}=0.1$. Observe that the Young modulus is very large so that the continuous and discrete functionals are expected to be convex. The analytical solution is chosen to be in $H^{3}(\Omega)(n=4)$. The results are summarized in Tables 1 and 2. Optimal convergence rates are observed in both cases. In the $\mathbb{P}_{1} / \mathbb{P}_{0}$ case, this result matches the estimate of Theorem 4.9. In the $\mathbb{P}_{2} / \mathbb{P}_{1}$ case, the numerical result improves on the estimate of Theorem 4.11 which is suboptimal. Moreover, in both cases, the Lagrange multiplier super-converges although in the present setting for $V_{h}$, the discrete inf-sup condition is not robust with respect to mesh-size.

We now address the convergence of the decomposed Uzawa algorithm. We consider that convergence is reached when the difference between the exact solution and the approximate solution is below a given tolerance. The simulations are performed with $V_{h}=\mathcal{P}_{\mathrm{c}}^{1}\left(\mathcal{T}_{h}\right)$ and $M_{h}=\Lambda_{h}=\mathcal{P}_{\mathrm{d}}^{0}\left(\mathcal{F}_{h}\right)$. The numbers of iterations to reach convergence for various couples of parameters $(r, \rho)$ are collected in Table 3 . The two criteria $r \geq \rho$ and $r>2 k_{\psi^{\prime}}$ derived in Proposition 5.2 appear to be rather sharp.

Remark 6.1. Numerical results with Newton's method and the choice $V_{h}=\mathcal{P}_{\mathrm{c}}^{2}\left(\mathcal{T}_{h}\right), M_{h}=\mathcal{P}_{\mathrm{d}}^{1}\left(\mathcal{F}_{h}\right)$, and $\Lambda_{h}=\mathcal{P}_{\mathrm{c}}^{1}\left(\mathcal{F}_{h}\right)$ are presented in [25], where optimal convergence rates are reported. 


\section{A. Proof of Lemma 4.7}

Proof. Let $y_{h}:=\left(v_{h}, q_{h}\right) \in K_{h} \cap \operatorname{ker} \tilde{B}_{h}^{\sharp}$. Since ker $\tilde{B}_{h}^{\sharp} \subset \operatorname{ker} \tilde{B}_{h}$ (because $\left.\Lambda_{h} \subset M_{h}\right), y_{h} \in \operatorname{ker} \tilde{B}_{h}$. As a result, it is inferred from (4.20)-(4.22) that

$$
\begin{aligned}
0 & \leq\left\langle J_{r, h}^{\prime}\left(x_{h}\right), y_{h}-x_{h}\right\rangle_{Z^{\prime}, Z}+\left(\theta_{h}, \tilde{B}_{h}\left(y_{h}-x_{h}\right)\right)_{M} \\
& =\left\langle J_{r, h}^{\prime}\left(x_{h}\right), y_{h}-x_{h}\right\rangle_{Z^{\prime}, Z}
\end{aligned}
$$

Set $\delta_{h}=x_{h}-y_{h}:=\left(\delta_{h}^{v}, \delta_{h}^{p}\right)$ and observe that $\delta_{h} \in \operatorname{ker} \tilde{B}_{h}$. Then, since $y_{h} \in \operatorname{ker} \tilde{B}_{h}^{\sharp}$,

$$
\begin{aligned}
\left\langle J_{r, h}^{\prime}\left(x_{h}\right)-J_{r, h}^{\prime}\left(y_{h}\right), \delta_{h}\right\rangle_{Z^{\prime}, Z} & \leq\left\langle-J_{r, h}^{\prime}\left(y_{h}\right), \delta_{h}\right\rangle_{Z^{\prime}, Z}=\left\langle-J_{0, h}^{\prime}\left(y_{h}\right), \delta_{h}\right\rangle_{Z^{\prime}, Z} \\
& =\left\langle-J_{0}^{\prime}\left(y_{h}\right), \delta_{h}\right\rangle_{Z^{\prime}, Z}+\left\langle J_{0}^{\prime}\left(y_{h}\right)-J_{0, h}^{\prime}\left(y_{h}\right), \delta_{h}\right\rangle_{Z^{\prime}, Z} \\
& =\left\langle-J_{0}^{\prime}(x), \delta_{h}\right\rangle_{Z^{\prime}, Z}+\left\langle J_{0}^{\prime}(x)-J_{0}^{\prime}\left(y_{h}\right), \delta_{h}\right\rangle_{Z^{\prime}, Z}+\left\langle J_{0}^{\prime}\left(y_{h}\right)-J_{0, h}^{\prime}\left(y_{h}\right), \delta_{h}\right\rangle_{Z^{\prime}, Z} \\
& =\left\langle-J_{0}^{\prime}(x), \delta_{h}\right\rangle_{Z^{\prime}, Z}+\left\langle J_{0}^{\prime}(x)-J_{0}^{\prime}\left(y_{h}\right), \delta_{h}\right\rangle_{Z^{\prime}, Z}+\left(\psi^{\prime}\left(q_{h}\right), \delta_{h}^{q}\right)_{M}-\left(\psi^{\prime}\left(q_{h}\right), \delta_{h}^{q}\right)_{M_{h}} \\
& \lesssim\left\langle-J_{0}^{\prime}(x), \delta_{h}\right\rangle_{Z^{\prime}, Z}+\left(\left\|x-y_{h}\right\|_{Z}+\eta_{\text {quad }}\left(q_{h}\right)\right)\left\|\delta_{h}\right\|_{Z},
\end{aligned}
$$

using the Lipschitz-continuity of $J_{0}^{\prime}$ and the definition (4.30). Focusing on the first term in the right-hand side, we observe that for all $y:=(v, q) \in K$,

$$
\begin{aligned}
\left\langle-J_{0}^{\prime}(x), \delta_{h}\right\rangle_{Z^{\prime}, Z} & =\left\langle-J_{0}^{\prime}(x), y-y_{h}\right\rangle_{Z^{\prime}, Z}+\left\langle-J_{0}^{\prime}(x), x_{h}-y\right\rangle_{Z^{\prime}, Z} \\
& =\left\langle-J_{0}^{\prime}(x), y-y_{h}\right\rangle_{Z^{\prime}, Z}+\left(\theta, \tilde{B}\left(x_{h}-y\right)\right)_{M}-\left\langle\partial_{y} L_{0}(x, \theta), x_{h}-y\right\rangle_{Z^{\prime}, Z} \\
& =\left\langle-J_{0}^{\prime}(x), y-x\right\rangle_{Z^{\prime}, Z}+\left\langle-J_{0}^{\prime}(x), x-y_{h}\right\rangle_{Z^{\prime}, Z}+\left(\theta, \tilde{B}\left(x_{h}-y\right)\right)_{M}-\left\langle\partial_{y} L_{0}(x, \theta), x_{h}-y\right\rangle_{Z^{\prime}, Z} \\
& \leq\left(\theta, \tilde{B}\left(x_{h}-x\right)\right)_{M}+\left\langle-J_{0}^{\prime}(x), x-y_{h}\right\rangle_{Z^{\prime}, Z}-\left\langle\partial_{y} L_{0}(x, \theta), x_{h}-y\right\rangle_{Z^{\prime}, Z} \\
& =\left(\theta, \tilde{B}\left(x_{h}-y_{h}\right)\right)_{M}-\left\langle\partial_{y} L_{0}(x, \theta), x-y_{h}\right\rangle_{Z^{\prime}, Z}-\left\langle\partial_{y} L_{0}(x, \theta), x_{h}-y\right\rangle_{Z^{\prime}, Z},
\end{aligned}
$$

where we have used the fact that $\left\langle-J_{0}^{\prime}(x), y-x\right\rangle_{Z^{\prime}, Z} \leq(\theta, \tilde{B}(y-x))_{M}$ since $(x, \theta)$ is the exact solution and $y \in K$. Furthermore, using the definitions (4.28) and (4.29) and the fact that $V_{h} \subset V$, it is readily seen that

$$
\left\langle\partial_{y} L_{0}(x, \theta), y_{h}-x\right\rangle_{Z^{\prime}, Z}=\left(\psi^{\prime}(p)-\theta, q_{h}-p\right)_{M}=\eta_{\text {unil }}\left(q_{h}\right),
$$

and

so that

$$
\left\langle\partial_{y} L_{0}(x, \theta), y-x_{h}\right\rangle_{Z^{\prime}, Z}=\left(\psi^{\prime}(p)-\theta, q-p_{h}\right)_{M}=\eta_{\text {unil }}(q),
$$

$$
\left\langle-J_{0}^{\prime}(x), \delta_{h}\right\rangle_{Z^{\prime}, Z} \leq\left(\theta, \tilde{B}\left(x_{h}-y_{h}\right)\right)_{M}+\eta_{\text {unil }}\left(q_{h}\right)+\eta_{\text {unil }}(q) .
$$

Since $H^{+}$is dense in $M^{+}$, the above estimate can be extended by continuity to $q \in M^{+}$. Furthermore, observing that $\left(x_{h}-y_{h}\right) \in \operatorname{ker} \tilde{B}_{h}$, there holds

$$
\begin{aligned}
\left\langle-J_{0}^{\prime}(x), \delta_{h}\right\rangle_{Z^{\prime}, Z} & \leq\left(\theta-\Pi_{\Lambda_{h}} \theta, \tilde{B}\left(x_{h}-y_{h}\right)\right)_{M}+\eta_{\text {unil }}\left(q_{h}\right)+\eta_{\text {unil }}(q) \\
& \leq\left\|\theta-\Pi_{\Lambda_{h}} \theta\right\|_{M}\left\|\tilde{B}\left(x_{h}-y_{h}\right)\right\|_{M}+\eta_{\text {unil }}\left(q_{h}\right)+\eta_{\text {unil }}(q) .
\end{aligned}
$$

Moreover, if $\Lambda_{h}=M_{h}$,

$$
\left\|\tilde{B}\left(x_{h}-y_{h}\right)\right\|_{M}=\left\|\left(I-\Pi_{\Lambda_{h}}\right) B\left(u_{h}-v_{h}\right)\right\|_{M} \lesssim h^{1 / 2}\left\|B\left(u_{h}-v_{h}\right)\right\|_{H} \lesssim h^{1 / 2}\left\|u_{h}-v_{h}\right\|_{V},
$$

while $\left\|\tilde{B}\left(x_{h}-y_{h}\right)\right\|_{M} \lesssim\left\|x_{h}-y_{h}\right\|_{Z}$ if $\Lambda_{h} \neq M_{h}$. Thus, in all cases, $\left\|\tilde{B}\left(x_{h}-y_{h}\right)\right\|_{M} \lesssim h^{s / 2}\left\|x_{h}-y_{h}\right\|_{Z}$ with $s=1$ if $\Lambda_{h}=M_{h}$ and $s=0$ if $\Lambda_{h} \neq M_{h}$, and this yields

$$
\left\langle-J_{0}^{\prime}(x), \delta_{h}\right\rangle_{Z^{\prime}, Z} \lesssim h^{s / 2}\left\|\theta-\Pi_{\Lambda_{h}} \theta\right\|_{M}\left\|x_{h}-y_{h}\right\|_{Z}+\eta_{\text {unil }}\left(q_{h}\right)+\eta_{\text {unil }}(q) .
$$


Collecting the above estimates and using (4.19) together with a Young inequality, it is inferred that

$$
\left\|x_{h}-y_{h}\right\|_{Z}^{2} \lesssim\left\|x-y_{h}\right\|_{Z}^{2}+\eta_{\text {unil }}\left(q_{h}\right)+\eta_{\text {quad }}\left(q_{h}\right)^{2}+\eta_{\text {unil }}(q)+h^{s}\left\|\theta-\Pi_{\Lambda_{h}} \theta\right\|_{M}^{2},
$$

whence the bound on $\left\|x-x_{h}\right\|_{Z}$ follows using a triangle inequality. Finally, to derive the bound on $h^{1 / 2}\left\|\theta-\theta_{h}\right\|_{M}$, observe that for $v_{h} \in V_{h}$ and since $V_{h} \subset V$,

$$
\begin{aligned}
\left(\theta_{h}-\Pi_{\Lambda_{h}} \theta, B_{h} v_{h}\right)_{M} & =\left(\theta_{h}-\Pi_{\Lambda_{h}} \theta, B v_{h}\right)_{M} \\
& =\left(\theta_{h}-\theta, B v_{h}\right)_{M}+\left(\theta-\Pi_{\Lambda_{h}} \theta, B v_{h}\right)_{M} \\
& =\left\langle W^{\prime}(u)-W^{\prime}\left(u_{h}\right), v_{h}\right\rangle_{V^{\prime}, V}+r\left(\tilde{B}_{h}^{\sharp}\left(u-u_{h}\right), B v_{h}\right)_{M}+\left(\theta-\Pi_{\Lambda_{h}} \theta, B v_{h}-\Pi_{\Lambda_{h}} B v_{h}\right)_{M} \\
& \lesssim\left\|x-x_{h}\right\|_{Z}\left\|v_{h}\right\|_{V}+\left\|\theta-\Pi_{\Lambda_{h}} \theta\right\|_{M}\left\|B v_{h}-\Pi_{\Lambda_{h}} B v_{h}\right\|_{M} \\
& \lesssim\left(\left\|x-x_{h}\right\|_{Z}+h^{1 / 2}\left\|\theta-\Pi_{\Lambda_{h}} \theta\right\|_{M}\right)\left\|v_{h}\right\|_{V},
\end{aligned}
$$

whence the desired estimate results from the discrete inf-sup condition and a triangle inequality.

\section{B. Proof of Proposition 5.2}

Proof. The couple $\left(u_{h}, p_{h}\right) \in V_{h} \times M_{h}^{+}$is such that

$$
\begin{array}{ll}
\left\langle W^{\prime}\left(u_{h}\right), v_{h}\right\rangle_{V^{\prime}, V}+\left(\theta_{h}, B v_{h}\right)_{M}+r\left(B u_{h}-p_{h}, B v_{h}\right)_{M}=0, & \forall v_{h} \in V_{h}, \\
\left(\psi^{\prime}\left(p_{h}\right), q_{h}-p_{h}\right)_{M_{h}}-\left(\theta_{h}, q_{h}-p_{h}\right)_{M}-r\left(B u_{h}-p_{h}, q_{h}-p_{h}\right)_{M} \geq 0, & \forall q_{h} \in M_{h}^{+}, \\
\theta_{h}=\theta_{h}+\rho\left(\Pi_{M_{h}} B u_{h}-p_{h}\right) . &
\end{array}
$$

The couple $\left(u_{h}^{n}, p_{h}^{n}\right) \in V_{h} \times M_{h}^{+}$is such that

$$
\begin{array}{ll}
\left\langle W^{\prime}\left(u_{h}^{n}\right), v_{h}\right\rangle_{V^{\prime}, V}+\left(\theta_{h}^{n-1}, B v_{h}\right)_{M}+r\left(B u_{h}^{n}-p_{h}^{n-1}, B v_{h}\right)_{M}=0, & \forall v_{h} \in V_{h}, \\
\left(\psi^{\prime}\left(p_{h}^{n}\right), q_{h}-p_{h}^{n}\right)_{M_{h}}-\left(\theta_{h}^{n-1}, q_{h}-p_{h}^{n}\right)_{M}-r\left(B u_{h}^{n}-p_{h}^{n}, q_{h}-p_{h}^{n}\right)_{M} \geq 0, & \forall q_{h} \in M_{h}^{+}, \\
\theta_{h}^{n}=\theta_{h}^{n-1}+\rho\left(\Pi_{M_{h}} B u_{h}^{n}-p_{h}^{n}\right) &
\end{array}
$$

Set $\bar{\theta}_{h}^{n}:=\theta_{h}^{n}-\theta_{h}, \bar{p}_{h}^{n}:=p_{h}^{n}-p_{h}$, and $\bar{u}_{h}^{n}:=u_{h}^{n}-u_{h}$. Applying (B.1) and (B.4) to $v_{h}=\bar{u}_{h}^{n}$ and subtracting yields

$$
\left\langle W^{\prime}\left(\bar{u}_{h}^{n}\right), \bar{u}_{h}^{n}\right\rangle_{V^{\prime}, V}+\left(\bar{\theta}_{h}^{n-1}, B \bar{u}_{h}^{n}\right)_{M}+r\left\|B \bar{u}_{h}^{n}\right\|_{M}^{2}-r\left(\bar{p}_{h}^{n-1}, B \bar{u}_{h}^{n}\right)_{M}=0,
$$

where the linearity of $W^{\prime}$ has been used. Similarly, using (B.2) with $q_{h}=p_{h}^{n}$ and (B.5) with $q_{h}=p_{h}$ and subtracting leads to

$$
\left(\psi^{\prime}\left(p_{h}^{n}\right)-\psi^{\prime}\left(p_{h}\right), p_{h}^{n}-p_{h}\right)_{M_{h}}-\left(\bar{\theta}_{h}^{n-1}, \bar{p}_{h}^{n}\right)_{M}+r\left\|\bar{p}_{h}^{n}\right\|_{M}^{2}-r\left(\bar{p}_{h}^{n}, B \bar{u}_{h}^{n}\right)_{M} \leq 0 .
$$

Adding (B.7) and (B.8) and setting $\alpha:=\alpha_{W}-2 k_{\psi^{\prime}} c_{M}^{2} c_{B}^{2}$ and $\beta:=r-2 k_{\psi^{\prime}}$ yields

$$
\begin{aligned}
-\left(\bar{\theta}_{h}^{n-1}, B \bar{u}_{h}^{n}-\bar{p}_{h}^{n}\right)_{M} \geq & \left\langle W^{\prime}\left(\bar{u}_{h}^{n}\right), \bar{u}_{h}^{n}\right\rangle_{V^{\prime}, V}+\left(\psi^{\prime}\left(p_{h}^{n}\right)-\psi^{\prime}\left(p_{h}\right), p_{h}^{n}-p_{h}\right)_{M_{h}} \\
& +r\left\|B \bar{u} \bar{p}_{h}^{n}-\bar{p}_{h}^{n}\right\|_{M}^{2}+r\left(\bar{p}_{h}^{n}-\bar{p}_{h}^{n-1}, B \bar{u}_{h}^{n}\right)_{M} \\
\geq & \alpha_{W}\left\|\bar{u}_{h}^{n}\right\|_{V}^{2}-k_{\psi^{\prime}}\left\|\bar{p}_{h}^{n}\right\|_{M}^{2}+r\left\|B \bar{u}_{h}^{n}-\bar{p}_{h}^{n}\right\|_{M}^{2}+r\left(\bar{p}_{h}^{n}-\bar{p}_{h}^{n-1}, B \bar{u}_{h}^{n}\right)_{M} \\
\geq & \alpha_{W}\left\|\bar{u}_{h}^{n}\right\|_{V}^{2}-2 k_{\psi^{\prime}}\left\|B \bar{u}_{h}^{n}\right\|_{M}^{2}-2 k_{\psi^{\prime}}\left\|B \bar{u}_{h}^{n}-\bar{p}_{h}^{n}\right\|_{M}^{2}+r\left\|B \bar{u}_{h}^{n}-\bar{p}_{h}^{n}\right\|_{M}^{2}+r\left(\bar{p}_{h}^{n}-\bar{p}_{h}^{n-1}, B \bar{u}_{h}^{n}\right)_{M} \\
\geq & \alpha\left\|\bar{u}_{h}^{n}\right\|_{V}^{2}+\beta\left\|B \bar{u}_{h}^{n}-\bar{p}_{h}^{n}\right\|_{M}^{2}+r\left(\bar{p}_{h}^{n}-\bar{p}_{h}^{n-1}, B \bar{u}_{h}^{n}\right)_{M} .
\end{aligned}
$$


Adding (B.5) written at iteration $n-1$ with $q_{h}=p_{h}^{n}$ and (B.5) written at iteration $n$ with $q_{h}=p_{h}^{n-1}$ and since $\bar{p}_{h}^{n}-\bar{p}_{h}^{n-1}=p_{h}^{n}-p_{h}^{n-1}$ and so on, yields

$$
\begin{aligned}
-\left(\bar{\theta}_{h}^{n-2}-\bar{\theta}_{h}^{n-1}, \bar{p}_{h}^{n}-\bar{p}_{h}^{n-1}\right)_{M} \geq & \left(\psi^{\prime}\left(p_{h}^{n}\right)-\psi^{\prime}\left(p_{h}^{n-1}\right), p_{h}^{n}-p_{h}^{n-1}\right)+r\left\|\bar{p}_{h}^{n}-\bar{p}_{h}^{n-1}\right\|_{M}^{2} \\
& -r\left(\bar{p}_{h}^{n}-\bar{p}_{h}^{n-1}, B \bar{u}_{h}^{n}-B \bar{u}_{h}^{n-1}\right)_{M} \\
\geq & \left(r-k_{\psi^{\prime}}\right)\left\|\bar{p}_{h}^{n}-\bar{p}_{h}^{n-1}\right\|_{M}^{2}-r\left(\bar{p}_{h}^{n}-\bar{p}_{h}^{n-1}, B \bar{u}_{h}^{n}-B \bar{u}_{h}^{n-1}\right)_{M} .
\end{aligned}
$$

Owing to (B.3) and (B.6) at iteration $(n-1)$,

$$
\bar{\theta}_{h}^{n-1}=\bar{\theta}_{h}^{n-2}+\rho\left(\Pi_{M_{h}} B \bar{u}_{h}^{n-1}-\bar{p}_{h}^{n-1}\right),
$$

whence, owing to (B.10),

$$
r\left(\bar{p}_{h}^{n}-\bar{p}_{h}^{n-1}, B \bar{u}_{h}^{n}-B \bar{u}_{h}^{n-1}\right)_{M} \geq \beta\left\|\bar{p}_{h}^{n}-\bar{p}_{h}^{n-1}\right\|_{M}^{2}-\rho\left(B \bar{u}_{h}^{n-1}-\bar{p}_{h}^{n-1}, \bar{p}_{h}^{n}-\bar{p}_{h}^{n-1}\right)_{M} .
$$

This equation can be reorganized as

$$
r\left(B \bar{u}_{h}^{n}, \bar{p}_{h}^{n}-\bar{p}_{h}^{n-1}\right)_{M} \geq \beta\left\|\bar{p}_{h}^{n}-\bar{p}_{h}^{n-1}\right\|_{M}^{2}+(r-\rho)\left(B \bar{u}_{h}^{n-1}-\bar{p}_{h}^{n-1}, \bar{p}_{h}^{n}-\bar{p}_{h}^{n-1}\right)_{M}+r\left(\bar{p}_{h}^{n-1}, \bar{p}_{h}^{n}-\bar{p}_{h}^{n-1}\right)_{M} .
$$

Using the identity $2\left(\bar{p}_{h}^{n-1}, \bar{p}_{h}^{n}-\bar{p}_{h}^{n-1}\right)_{M}=\left\|\bar{p}_{h}^{n}\right\|_{M}^{2}-\left\|\bar{p}_{h}^{n-1}\right\|_{M}^{2}-\left\|\bar{p}_{h}^{n}-\bar{p}_{h}^{n-1}\right\|_{M}^{2}$, we turn (B.12) into

$$
\begin{aligned}
2 r\left(B \bar{u}_{h}^{n}, \bar{p}_{h}^{n}-\bar{p}_{h}^{n-1}\right)_{M} \geq & (2 \beta-r)\left\|\bar{p}_{h}^{n}-\bar{p}_{h}^{n-1}\right\|_{M}^{2}+r\left(\left\|\bar{p}_{h}^{n}\right\|_{M}^{2}-\left\|\bar{p}_{h}^{n-1}\right\|_{M}^{2}\right) \\
& +2(r-\rho)\left(B \bar{u}_{h}^{n-1}-\bar{p}_{h}^{n-1}, \bar{p}_{h}^{n}-\bar{p}_{h}^{n-1}\right)_{M} .
\end{aligned}
$$

Squaring (B.11) at iteration $n$ leads to

$$
\left\|\bar{\theta}_{h}^{n-1}\right\|_{M}^{2}-\left\|\bar{\theta}_{h}^{n}\right\|_{M}^{2} \geq-2 \rho\left(\bar{\theta}_{h}^{n-1}, B \bar{u}_{h}^{n}-\bar{p}_{h}^{n}\right)_{M}-\rho^{2}\left\|B \bar{u}_{h}^{n}-\bar{p}_{h}^{n}\right\|_{M}^{2} .
$$

Finally, collecting (B.9) and (B.13) and using $r \geq \rho$ yields

$$
\begin{gathered}
\left\|\bar{\theta}_{h}^{n-1}\right\|_{M}^{2}-\left\|\bar{\theta}_{h}^{n}\right\|_{M}^{2} \geq \\
\quad 2 \rho \alpha\left\|\bar{u}_{h}^{n}\right\|_{V}^{2}+\rho(2 \beta-\rho)\left\|B \bar{u}_{h}^{n}-\bar{p}_{h}^{n}\right\|_{M}^{2}+\rho(2 \beta-r)\left\|\bar{p}_{h}^{n}-\bar{p}_{h}^{n-1}\right\|_{M}^{2} \\
+r \rho\left(\left\|\bar{p}_{h}^{n}\right\|_{M}^{2}-\left\|\bar{p}_{h}^{n-1}\right\|_{M}^{2}\right)+2 \rho(r-\rho)\left(B \bar{u}_{h}^{n-1}-\bar{p}_{h}^{n-1}, \bar{p}_{h}^{n}-\bar{p}_{h}^{n-1}\right)_{M} \\
\Leftrightarrow\left(\left\|\bar{\theta}_{h}^{n-1}\right\|_{M}^{2}+r \rho\left\|\bar{p}_{h}^{n-1}\right\|_{M}^{2}\right)-\left(\left\|\bar{\theta}_{h}^{n}\right\|_{M}^{2}+r \rho\left\|\bar{p}_{h}^{n}\right\|_{M}^{2}\right) \geq 2 \rho \alpha\left\|\bar{u}_{h}^{n}\right\|_{V}^{2}+\rho(2 \beta-\rho)\left\|B \bar{u}_{h}^{n}-\bar{p}_{h}^{n}\right\|_{M}^{2} \\
+\rho(2 \beta-r)\left\|\bar{p}_{h}^{n}-\bar{p}_{h}^{n-1}\right\|_{M}^{2}+2 \rho(r-\rho)\left(B \bar{u}_{h}^{n-1}-\bar{p}_{h}^{n-1}, \bar{p}_{h}^{n}-\bar{p}_{h}^{n-1}\right)_{M} \\
\Rightarrow\left(\left\|\bar{\theta}_{h}^{n-1}\right\|_{M}^{2}+r \rho\left\|\bar{p}_{h}^{n-1}\right\|_{M}^{2}\right)-\left(\left\|\bar{\theta}_{h}^{n}\right\|_{M}^{2}+r \rho\left\|_{h}^{n}\right\|_{M}^{2}\right) \geq 2 \rho \alpha\left\|\bar{u}_{h}^{n}\right\|_{V}^{2}+\rho(2 \beta-\rho)\left\|B \bar{u}_{h}^{n}-\bar{p}_{h}^{n}\right\|_{M}^{2} \\
\quad+\rho(2 \beta-r)\left\|\bar{p}_{h}^{n}-\bar{p}_{h}^{n-1}\right\|_{M}^{2}-\rho(r-\rho)\left(\left\|B \bar{u}_{h}^{n-1}-\bar{p}_{h}^{n-1}\right\|_{M}^{2}+\left\|\bar{p}_{h}^{n}-\bar{p}_{h}^{n-1}\right\|_{M}^{2}\right) .
\end{gathered}
$$

Set $\epsilon^{n}=\left\|\bar{\theta}_{h}^{n}\right\|_{M}^{2}+r \rho\left\|\bar{p}_{h}^{n}\right\|_{M}^{2}+\rho(r-\rho)\left\|B \bar{u}_{h}^{n}-\bar{p}_{h}^{n}\right\|_{M}^{2}$ so that (B.14) can be rewritten as

$$
\epsilon^{n-1}-\epsilon^{n} \geq 2 \rho \alpha\left\|\bar{u}_{h}^{n}\right\|_{V}^{2}+\rho(2 \beta-r)\left\|B \bar{u}_{h}^{n}-\bar{p}_{h}^{n}\right\|_{M}^{2}+\rho(2 \beta-2 r+\rho)\left\|\bar{p}_{h}^{n}-\bar{p}_{h}^{n-1}\right\|_{M}^{2} .
$$

By assumption, $\alpha>0,2 \beta-r>0$, and $2 \beta-2 r+\rho=\rho-4 k_{\psi^{\prime}} \geq 0$. Hence, the sequence $\left(\epsilon^{n}\right)_{n \in \mathbb{N}}$, which is clearly nonnegative, is decreasing; thus it converges. As a consequence, the right-hand side of (B.15) converges to zero, implying that the sequences $\left(u_{h}^{n}\right)_{n \in \mathbb{N}}$ and $\left(p_{h}^{n}\right)_{n \in \mathbb{N}}$ converge to $u_{h}$ and $p_{h}$ respectively. Finally, the sequence $\left(\theta_{h}^{n}\right)_{n \in \mathbb{N}}$ is bounded. According to the Bolzano-Weierstrass theorem, there exists a converging subsequence, and its limit is easily seen to be $\theta_{h}$. By uniqueness of the discrete solution $\left(u_{h}, p_{h}, \theta_{h}\right)$, the whole sequence $\left(\theta_{h}^{n}\right)_{n \in \mathbb{N}}$ converges to $\theta_{h}$. 


\section{REFERENCES}

[1] P. Alart and A. Curnier, A mixed formulation for frictional contact problems prone to Newton like solution methods. Comput. Methods Appl. Mech. Engrg. 92 (1991) 353-375.

[2] K.J. Bathe and F. Brezzi, Stability of finite element mixed interpolations for contact problems. Atti Accad. Naz. Lincei Cl. Sci. Fis. Mat. Natur. Rend. Lincei (9) Mat. Appl. 12 (2001) 167-183.

[3] D.P. Bertsekas, Constrained Optimization and Lagrange Multiplier Methods. Athena Scientific (1982).

[4] D.P. Bertsekas, Nonlinear Programming. Athena Scientific (1999).

[5] B. Bourdin, G.A. Francfort and J.-J. Marigo, The variational approach to fracture. J. Elasticity 91 (2008) 5-148.

[6] L. Champaney, J.-Y. Cognard and P. Ladevèze, Modular analysis of assemblages of three-dimensional structures with unilateral contact conditions. Comput. Struct. 73 (1999) 249-266.

[7] Z. Chen, On the augmented Lagrangian approach to Signorini elastic contact problem. Numer. Math. 88 (2001) 641-659.

[8] P.G. Ciarlet, Mathematical elasticity, Vol. I: Three-dimensional elasticity, Studies in Mathematics and its Applications 20. North-Holland Publishing Co., Amsterdam (1988).

[9] F.H. Clarke, Optimization and nonsmooth analysis, Classics in Applied Mathematics 5. Society for Industrial and Applied Mathematics (SIAM), Philadelphia, USA, second edition (1990).

[10] Z. Denkowski, S. Migórski and N.S. Papageorgiou, An introduction to nonlinear analysis: applications. Kluwer Academic Publishers, Boston, USA (2003).

[11] I. Ekeland and R. Témam, Convex analysis and variational problems, Classics in Applied Mathematics. 28. Society for Industrial and Applied Mathematics (SIAM), Philadelphia, USA (1999).

[12] A. Ern and J.-L. Guermond, Theory and Practice of Finite Elements, Applied Mathematical Sciences 159. Springer-Verlag, New York, USA (2004).

[13] M. Fortin and R. Glowinski, Augmented Lagrangian methods: Applications to the numerical solution of boundary value problems, Studies in Mathematics and its Applications 15. North-Holland Publishing Co., Amsterdam (1983).

[14] M. Frémond, Contact with adhesion, in Topics in nonsmooth mechanics, Birkhäuser, Basel, Switzerland (1988) 157-185.

[15] R. Glowinski and P. Le Tallec, Augmented Lagrangian and operator-splitting methods in nonlinear mechanics, SIAM Studies in Applied Mathematics 9. Society for Industrial and Applied Mathematics (SIAM), Philadelphia, USA (1989).

[16] J. Haslinger, I. Hlaváček and J. Nečas, Numerical methods for unilateral problems in solid mechanics, in Handbook of numerical analysis IV, Amsterdam, North-Holland (1996) 313-485.

[17] P. Hauret and P. Le Tallec, A discontinuous stabilized mortar method for general 3d elastic problems. Comput. Methods Appl. Mech. Engrg. 196 (2007) 4881-4900.

[18] P. Hild and P. Laborde, Quadratic finite element methods for unilateral contact problems. Appl. Numer. Math. 41 (2002) 401-421.

[19] S. Hüeber and B.I. Wohlmuth, An optimal a priori error estimate for nonlinear multibody contact problems. SIAM J. Numer. Anal. 43 (2005) 156-173 (electronic).

[20] N. Kikuchi and J.T. Oden, Contact problems in elasticity: a study of variational inequalities and finite element methods, SIAM Studies in Applied Mathematics 8. Society for Industrial and Applied Mathematics (SIAM), Philadelphia, USA (1988).

[21] D. Kinderlehrer, Remarks about Signorini's problem in linear elasticity. Ann. Scuola Norm. Sup. Pisa Cl. Sci. (4) 8 (1981) 605-645.

[22] K. Kunisch and G. Stadler, Generalized Newton methods for the 2D-Signorini contact problem with friction in function space. ESAIM: M2AN 39 (2005) 827-854.

[23] P. Ladevèze, Nonlinear Computational Structural Mechanics - New Approaches and Non-Incremental Methods of Calculation. Springer-Verlag (1999).

[24] J.-L. Lions and E. Magenes, Non-homogeneous boundary value problems and applications I, Die Grundlehren der mathematischen Wissenschaften, Band 181. Springer-Verlag, New York, USA (1972).

[25] E. Lorentz, A mixed interface finite element for cohesive zone models. Comput. Methods Appl. Mech. Engrg. 198 (2008) 302-317.

[26] M. Marcus and V.J. Mizel, Every superposition operator mapping one Sobolev space into another is continuous. J. Funct. Anal. 33 (1979) 217-229.

[27] M. Moussaoui and K. Khodja, Régularité des solutions d'un problème mêlé Dirichlet-Signorini dans un domaine polygonal plan. Commun. Partial Differ. Equ. 17 (1992) 805-826.

[28] L.. Qi and J. Sun, A nonsmooth version of Newton's method. Math. Program. 58 (1993) 353-367.

[29] L. Slimane, A. Bendali and P. Laborde, Mixed formulations for a class of variational inequalities. ESAIM: M2AN 38 (2004) $177-201$. 